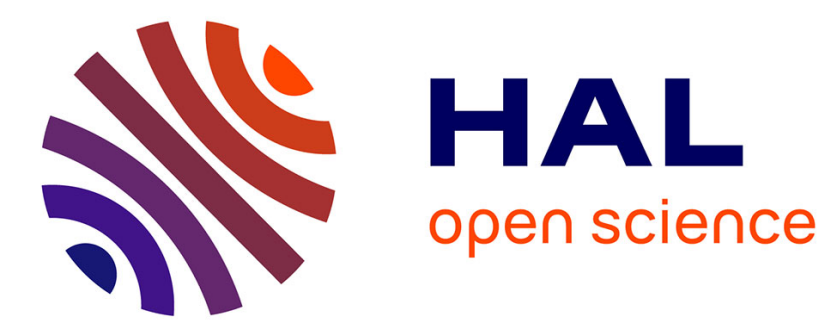

\title{
Modélisation des propriétés électriques des décharges RF diode
}

\author{
A.M. Pointu
}

\section{To cite this version:}

A.M. Pointu. Modélisation des propriétés électriques des décharges RF diode. Revue de Physique Appliquée, 1989, 24 (3), pp.257-276. 10.1051/rphysap:01989002403025700 . jpa-00246048

\section{HAL Id: jpa-00246048 https://hal.science/jpa-00246048}

Submitted on 1 Jan 1989

HAL is a multi-disciplinary open access archive for the deposit and dissemination of scientific research documents, whether they are published or not. The documents may come from teaching and research institutions in France or abroad, or from public or private research centers.
L'archive ouverte pluridisciplinaire HAL, est destinée au dépôt et à la diffusion de documents scientifiques de niveau recherche, publiés ou non, émanant des établissements d'enseignement et de recherche français ou étrangers, des laboratoires publics ou privés. 


\title{
Modélisation des propriétés électriques des décharges RF diode
}

\author{
A. M. Pointu \\ Laboratoire de Physique des Gaz et des Plasmas (*), Université Paris Sud, 91405 Orsay, Cedex, France
}

(Reçu le 10 juin 1988, révisé le 4 octobre 1988, accepté le 24 octobre 1988)

\begin{abstract}
Résumé. - L'impédance des décharges RF est dominée par les gaines des électrodes. Jusqu'à un passé récent, ces gaines étaient assimilées à un circuit électrique sommaire constitué d'éléments invariables dans le temps. Aujourd'hui, des modèles plus élaborés existent, utilisant la bonne connaissance des sondes électrostatiques. Nous en présentons ici une synthèse mettant en évidence qu'une description non auto-cohérente très simple suffit pour déterminer l'évolution dans le temps des potentiels de gaines. Cette description implique cependant que la fréquence d'excitation soit très inférieure ou très supérieure à la fréquence plasma ionique et que les processus inélastiques dans les gaines soient négligeables. Sa comparaison à des résultats expérimentaux publiés est satisfaisante.
\end{abstract}

\begin{abstract}
The impedance of RF discharges is dominated by the electrode sheaths. Until recently, these sheaths were described by an equivalent electric circuit with constant elements. More accurate models are now available, using the good knowledge of the electrostatic probes. We present here a synthesis of such models. It demonstrates that a very simple non self consistent description is sufficient to determine the time evolution of the sheath potentials. It is assumed however that the excitation frequency is either lower or higher than the ionic plasma frequency, and that inelastic processes in the sheaths are negligible. It reasonably agrees with available experimental results.
\end{abstract}

\section{Introduction.}

Les décharges RF diode suscitent de l'intérêt pour leurs applications au traitement, au revêtement ou à la gravure de surfaces. Lorsque l'échantillon est porté par l'une des électrodes la connaissance aussi précise que possible de la région qui leur est adjacente est la condition nécessaire pour maîtriser les paramètres du processus et en particulier s'il s'agit de gravure, l'énergie des ions responsables de cette gravure. Cette énergie est, on le sait, déterminée par la chute de potentiel dans les gaines, gaines qui s'ajustent en fonction de la tension excitatrice de la décharge et constituent simultanément l'élément essentiel de son impédance.

L'étude des gaines des électrodes et celle du fonctionnement de la décharge sont donc indissociables. L'article qui va suivre a pour but de faire le point sur les modélisations de décharge RF. Jusqu'à un passé récent, en effet, elles reposaient sur un

$\left(^{*}\right)$ Unité de Recherche Associée au CNRS No. 073. circuit électrique équivalent, constitué d'éléments en parallèle constants dans le temps, capacité et diode [1], capacité, diode et résistance [2], résistance et capacité [3-5], résistance ou capacité $[4,6]$, ce dernier modèle étant une simplification du précédent par laquelle il est tenu compte du comportement dominant en fonction de la fréquence : résistif audessous du $\mathrm{MHz}$, capacitif à plus haute fréquence.

$A$ priori, de tels modèles ne peuvent décrire de manière satisfaisante le comportement essentiellement non linéaire des gaines. Or celui-ci est àssez bien connu par les théories nombreuses liées aux sondes électrostatiques. Complexes dans leur généralité, celles-ci se simplifient beaucoup en basse pression lorsque les sondes sont planes et de grande dimension, c'est-à-dire sans effets de bout, et lorsque le potentiel de sonde est très supérieur au potentiel thermique des électrons. Les expressions des courants en fonction des potentiels sont alors analytiques et aisément exploitables. Les modèles les plus récents des décharges $\mathrm{RF}$ se situent dans le cadre de cette approche [7] à [11]. Nous en présentons ici une 
synthèse et leur extension au cas collisionnel sans ionisation dans la gaine.

\section{Principes physiques du modèle.}

2.1 PASSAge D'Une décharge CONTINUE À UNE DÉCHARGE ALTERNATIVE. - Lorsque l'on applique entre les électrodes internes d'une décharge une différence de potentiel alternative, $V_{\mathrm{RF}} \cos \omega t$, le régime de fonctionnement dépend de la fréquence angulaire $\omega$. Si l'on fait varier celle-ci continûment à partir de zéro, on obtient d'abord un régime pour lequel, à chaque instant, la décharge se comporte comme une décharge continue, inversée à chaque demi-période. Pour des fréquences plus élevées, le système évolue ensuite progressivement vers une situation dans laquelle il n'y a plus de transport de charges d'une électrode à l'autre, lorsque leur excursion dans le champ électrique est inférieure à la distance interélectrodes. Alors disparaissent les notions d'anode et de cathode : la plupart des électrons oscillent sans être collectés et entretiennent la décharge en gagnant, de manière cumulative entre les chocs successifs sur les neutres, l'énergie cinétique nécessaire à l'ionisation. Le module moyen, $E_{0}$, du champ électrique alternatif dans le corps du plasma est celui qui règle la température électronique à une valeur assurant l'équilibre entre cette ionisation en volume et les pertes par recombinaison en volume ou sur les surfaces limitant la décharge. Hormis le cas où $\omega$ serait grand devant la fréquence de collision électron-neutre, il est donc du même ordre de grandeur que le champ électrique axial $X$ d'une colonne positive de même pression, $p$, ayant une dimension transverse égale à la dimension caractéristique, $d$, du système. Or, pour des valeurs du produit pd inférieures à 1 Torr.cm, $X d$ reste en général inférieur à $10 \mathrm{~V}$, ce qui est faible, comparé au potentiel $V_{\mathrm{RF}}$, lorsque ce dernier dépasse la centaine de volts. Par suite, le rôle des électrodes dans l'entretien de la décharge, bien qu'indispensable est quantitativement peu important par rapport à leur rôle de « paroi » : elles peuvent être considérées de manière approchée, comme des simples collecteurs de particules, comme si le plasma était créé par une source indépendante. De son côté, le plasma, bien que soumis au champ $E_{0} \cos \omega t$, est quasi équipotentiel au regard de l'échelle des tensions $V_{\mathrm{RF}}$.

2.2 HYPOTHÈSES DE BASE. - Les modèles qui vont être présentés utilisent le cadre de l'approche précédente. Ils sont donc basés sur deux premières hypothèses :

1) Le plasma est équipotentiel.

2) Les électrodes se comportent vis-à-vis du plasma comme des collecteurs passifs, ainsi que le feraient des sondes électrostatiques.
Ils supposent en outre que :

3) les électrons et les ions du plasma sont en équilibre maxwellien avec des températures respectives $T_{\mathrm{e}}$ et $T_{\mathrm{i}}\left(T_{\mathrm{e}} \gg T_{\mathrm{i}}\right)$ et des densités égales $n_{\mathrm{e}}=n_{\mathrm{i}}=n_{0}$.

4) Ces valeurs sont constantes sur une période, ce qui implique que soient constants les mécanismes de création et de perte de particules. En réalité, il se peut qu'une partie de l'ionisation soit due à l'émission secondaire des électrodes, émission qui est donc susceptible d'être modulée. Un tel effet ne sera pas considéré ici.

5) La décharge ne contient qu'une seule sorte d'ions de charge positive.

6) Eu égard à la surface généralement importante des électrodes, la variation de la surface d'entrée des gaines avec leur épaisseur est négligeable. L'hétérogénéité du plasma dans la direction radiale n'est pas non plus prise en compte. Les modèles mettront donc en jeu une seule dimension, $z$, suivant l'axe de la décharge.

7) A pression « élevée » comme à pression faible, l'ionisation dans les gaines est négligeable ainsi que l'échange de charge.

8) Enfin, ces gaines sont à charge d'espace positive, y compris celle de l'électrode la plus positive.

\section{Modèles d'une gaine ionique au voisinage d'une paroi polarisée.}

Soit

$$
V(z, t)=\bar{V}(z)+\tilde{V}(z, t)
$$

le potentiel négatif, mesuré par rapport au centre du plasma, au point $z$ et au temps $t$. Les symboles - et dénotent respectivement la valeur moyenne et la partie variable (à valeur moyenne nulle) de la fonction correspondante du temps. La fonction $\tilde{V}$ est périodique, de période $2 \pi / \omega$. La gaine s'étend entre sa lisière, en $z_{\mathrm{s}}\left(V=V_{\mathrm{s}}\right)$ et la paroi en $z=z_{w}\left(V=V_{w}\right)$. Le sens positif de l'axe $z$ est dirigé vers la paroi. A $V_{\mathrm{w}}$ supposé connu, nous nous proposons de déterminer le courant total de la paroi. Ce courant, $I$, est la superposition du courant de conduction

$$
I_{\mathrm{c}}=I_{\mathrm{i}}+I_{\mathrm{e}}
$$

somme algébrique des courants ioniques et électronique, respectivement $I_{\mathrm{i}}$ et $I_{\mathrm{e}}$, et du courant de déplacement

$$
I_{\mathrm{D}}=\left.\varepsilon_{0} A \frac{\mathrm{d}}{\mathrm{d} t} \frac{\partial|V|}{\partial z}\right|_{z_{w}}
$$

où $A$ est la surface de la paroi. Pour déterminer $I_{\mathrm{D}}$, le profil $V(z)$ doit être connu, ce qui implique que l'ensemble de la gaine ait été modélisé. Nous le ferons successivement pour une gaine statique puis modulée, en traitant à chaque fois les deux cas de la basse et de la moyenne pression. 
3.1 GAINE STATIQUE EN BASSE PRESSION. - Supposons que le potentiel de paroi soit constant et la pression suffisamment basse pour que les particules traversent la gaine sans effectuer de collision.

Ces électrons soumis au potentiel répulsif, $V$, se répartissent suivant la loi de Maxwell-Boltzmann

$$
\begin{aligned}
n_{\mathrm{e}}=n_{0} \exp ( & \left.-q|V| / k T_{\mathrm{e}}\right)= \\
& =n_{\mathrm{s}} \exp \left\{-q\left(|V|-\left|V_{\mathrm{s}}\right|\right) / k T_{\mathrm{e}}\right\}
\end{aligned}
$$

$n_{0}$ et $n_{\mathrm{s}}$ désignent la densité des électrons et des ions, respectivement dans le plasma et à la lisière de la gaine. $T_{\mathrm{e}}$ est la température électronique et $q$ la valeur absolue de la charge $q_{\mathrm{e}}$ d'un électron.

Le courant électrique sur la paroi est le flux thermique

$$
\left|I_{\mathrm{e}}\right|=n_{\mathrm{e} w} q A \sqrt{k T_{\mathrm{e}} / 2 \pi m_{\mathrm{e}}}
$$

où $m_{\mathrm{e}}$ est la masse d'un électron, soit, d'après la relation (4)

$$
\begin{aligned}
\left|I_{\mathrm{e}}\right|=n_{\mathrm{s}} q A & \sqrt{k T_{\mathrm{e}} / 2 \pi m_{\mathrm{e}}} \times \\
& \times \exp \left\{-q\left(\left|V_{w}\right|-\left|V_{\mathrm{s}}\right|\right) / k T_{\mathrm{e}}\right\} .
\end{aligned}
$$

Les ions de masse $m_{\mathrm{i}}$ et de charge $q_{\mathrm{i}}=q$ ont dans la gaine une vitesse dirigée supposée grande devant leur vitesse thermique

$$
v_{\mathrm{i}}=\left(2 q|V| / m_{\mathrm{i}}\right)^{1 / 2} .
$$

Leur flux reste constant à la traversée de la gaine et égal à sa valeur à la lisière

$$
n_{\mathrm{i}} v_{\mathrm{i}}=n_{\mathrm{s}} v_{\mathrm{s}} \text {. }
$$

On en déduit

$$
n_{\mathrm{i}}=n_{\mathrm{s}}\left(\left|V_{\mathrm{s}}\right| /|V|\right)^{1 / 2}
$$

Les relations (4) et (9) permettent d'écrire l'équation de Poisson sous la forme

$$
\begin{aligned}
\frac{\partial^{2}|V|}{\partial z^{2}}=\frac{n_{\mathrm{s}} q}{\varepsilon_{0}} & \left\{\left(\left|V_{\mathrm{s}}\right| /|V|\right)^{1 / 2}-\right. \\
& \left.-\exp \left[-q\left(|V|-\left|V_{\mathrm{s}}\right|\right) / k T_{\mathrm{e}}\right]\right\} .
\end{aligned}
$$

Le potentiel à la lisière de gaine doit satisfaire les deux conditions

$$
\begin{gathered}
\partial^{2}|V| / \partial z^{2}=0 \text { en } z=z_{\mathrm{s}} \\
(\partial / \partial z)\left(\partial^{2}|V| / \partial z^{2}\right)=0 \text { en } z=z_{\mathrm{s}} .
\end{gathered}
$$

La première définit la lisière comme étant la limite de la région à séparation de charge. La seconde implique qu'au-delà de cette limite, les densités électronique et ionique évoluent ensemble dans l'espace.

La résolution de l'équation (12) au moyen de la relation (10) conduit au critère de Bohm [12]

$$
\left|V_{\mathrm{s}}\right|=k T_{\mathrm{e}} / 2 q
$$

En l'introduisant dans les relations (4) et (9) on obtient finalement

$$
\begin{aligned}
& n_{\mathrm{s}}=0.6 n_{0} \\
& v_{\mathrm{s}}=\left(k T_{\mathrm{e}} / m_{\mathrm{i}}\right)^{1 / 2}
\end{aligned}
$$

d'où l'on peut déduire le courant de conduction ionique

$$
I_{\mathrm{i}}=A q n_{\mathrm{s}}\left(k T_{\mathrm{e}} / m_{\mathrm{i}}\right)^{1 / 2} .
$$

La connaissance du profil de potentiel implique l'intégration de l'équation (10), intégration qui est aisěe dans la limite de la gaine épaisse. Cette limite correspond en effet à une situation telle que l'on ait $n_{\mathrm{i}} \gg n_{\mathrm{e}}$ dans l'essentiel de la région comprise entre le point $z$ considéré et la lisière $z_{\mathrm{s}}$. Ceci se produit lorsque

$$
\left.\begin{array}{l}
|V| \gg\left|V_{\mathrm{s}}\right| \\
\partial|V| / \partial z \gg \partial|V| /\left.\partial z\right|_{z_{\mathrm{s}}}
\end{array}\right\}
$$

Avec les simplifications correspondantes de l'équation de départ et des conditions aux limites, on obtient la solution de Child Langmuir

$$
|V|=\frac{3}{4}\left(\sqrt{\frac{6 m_{\mathrm{i}}}{q}} \frac{I_{\mathrm{i}}}{A \varepsilon_{0}}\right)^{2 / 3}\left(z-z_{\mathrm{s}}\right)^{4 / 3} \text {. }
$$

3.2 GAINE STATIQUE EN HAUTE PRESSION SANS IONISATION. - Le potentiel de paroi étant encore ici constant, supposons que le mouvement des particules dans la gaine soit contrôlé par les collisions élastiques. Alors, le flux des particules d'espèces $j$ $(\mathrm{j}=\mathrm{i}, \mathrm{e})$ est une fonction du coefficient de diffusion, $D_{\mathrm{j}}$, et de la mobilité, $\mu_{\mathrm{j}}$, qui s'écrit

$$
n_{\mathrm{j}} v_{\mathrm{j}}=n_{\mathrm{j}} \mu_{\mathrm{j}} \partial|V| / \partial z-D_{\mathrm{j}} \partial n_{\mathrm{j}} / \partial z
$$

avec

$$
\mu_{\mathrm{j}}=q_{\mathrm{j}} / m_{\mathrm{j}} \nu_{\mathrm{jN}} \quad \text { et } \quad D_{\mathrm{j}}=k T_{\mathrm{j}} / m_{\mathrm{j}} \nu_{\mathrm{jN}} \text {. }
$$

$\nu_{\mathrm{jN}}$ représente la fréquence de collision pour l'échange de quantité de mouvement de la particule $\mathrm{j}$ avec les neutres.

Dans le cas des ions, le flux est dominé par la mobilité

$$
n_{\mathrm{i}} v_{\mathrm{i}} \simeq n_{\mathrm{i}} \mu_{\mathrm{i}} \partial|V| / \partial z
$$

Dans le cas des électrons, le potentiel est supposé assez répulsif pour que

$$
n_{\mathrm{e}} v_{\mathrm{e}} \ll n_{\mathrm{e}} \mu_{\mathrm{e}} \partial|V| / \partial z \simeq D_{\mathrm{e}} \partial n_{\mathrm{e}} / \partial z
$$

de sorte que la densité électronique se répartisse encore suivant la loi

$$
n_{\mathrm{e}}=n_{0} \exp \left(-q|V| / k T_{\mathrm{e}}\right) .
$$

En tout point où $n_{\mathrm{e}} \ll n_{\mathrm{i}}$, l'équation de Poisson prend la forme

$$
\partial^{2}|V| / \partial z^{2} \simeq n_{\mathrm{i}} q / \varepsilon_{0}
$$


soit, en raison de l'absence supposée d'ionisation dans la gaine et compte tenu de la relation (21)

$$
\partial^{2}|V| / \partial z^{2}=I_{\mathrm{i}} /\left(A \varepsilon_{0} \mu_{\mathrm{i}} \partial|V| / \partial z\right) .
$$

La mobilité ionique dépend, en général, de la valeur du champ électrique réduit, $E / p$, où $p$ est la pression. Elle est assez bien représentée par la relation

$$
\mu_{\mathrm{i}}=\frac{K}{p}\left(\frac{E}{p}\right)^{-\beta}
$$

où

$$
\begin{aligned}
& \beta=0 \text { pour } E / p<(E / p)_{\mathrm{L}} \\
& \beta=1 / 2 \text { pour } E / p>(E / p)_{\mathrm{L}} .
\end{aligned}
$$

La valeur limite $(E / p)_{\mathrm{L}}$ du champ réduit est typiquement de l'ordre de 10 à $100 \mathrm{~V} / \mathrm{cm}$.Torr. Après introduction de la relation (26) dans l'équation (25) l'intégration de celle-ci conduit à

$$
|V|=\frac{2}{3}\left(\frac{2 I_{\mathrm{i}}}{A \varepsilon_{0} \mu_{\mathrm{i}}}\right)^{1 / 2}\left(z-z_{\mathrm{s}}\right)^{3 / 2}
$$

pour les faibles champs réduits, et à

$$
|V|=\frac{3}{5}\left(\frac{3 I_{\mathrm{i}} \sqrt{p}}{2 A \varepsilon_{0} K}\right)^{2 / 3}\left(z-z_{\mathrm{s}}\right)^{5 / 3}
$$

pour les champs réduits « élevés ».

Les conditions aux limites qui ont été utilisées sont les mêmes que dans le cas de la chute libre (paragraphe 3.1).

Les relations (27) et (28) apparaissent comme des versions collisionnelles de la relation de Child Langmuir, dites «limitées par la mobilité ». Le flux d'ions donné par l'équation (21) est égal à sa valeur à la lisière de gaine. Il s'identifie en ce point au flux thermique, moyenné sur la distribution en énergie des ions, de tous ceux qui ont une vitesse positive et qui, en raison du potentiel attractif qu'ils vont ensuite subir, tomberont sur la paroi :

$$
n_{\mathrm{i}} v_{\mathrm{i}}=n_{\mathrm{s}}\left(k T_{\mathrm{i}} / 2 \pi m_{\mathrm{i}}\right)^{1 / 2}
$$

d'où

$$
I_{\mathrm{i}}=n_{\mathrm{s}} q A\left(k T_{\mathrm{i}} / 2 \pi m_{\mathrm{i}}\right)^{1 / 2}
$$

Le courant d'électrons, évalué sur la paroi, s'écrit

$$
\begin{array}{rl}
\left|I_{\mathrm{e}}\right|=2 n_{\mathrm{s}} & q A\left(k T_{\mathrm{e}} / 2 \pi m_{\mathrm{e}}\right)^{1 / 2} \times \\
& \times \exp \left[-q\left(\left|V_{w}\right|-\left|V_{\mathrm{s}}\right|\right) / k T_{\mathrm{e}}\right] .
\end{array}
$$

Cette expression a été calculée à partir de la densité à une distance de 1 libre parcours moyen de la paroi, $n_{\mathrm{e}}\left(z_{\mathrm{w}}-l\right)$; elle tient compte de l'effet de troncature de la fonction de distribution en ce point, qui introduit un facteur multiplicatif 2 lorsque $l \ll \sqrt{\mathrm{A}}[13]$.
3.3 DOMAINE DE VAlidité DES MODĖLES DE GAINE. - Les modèles développés aux paragraphes 3.1 et 3.2 correspondent à des cas limites de l'équation de conservation de la quantité de mouvement des ions

$$
\begin{aligned}
n_{\mathrm{i}} m_{\mathrm{i}} \frac{1}{2} \frac{\partial v_{\mathrm{i}}^{2}}{\partial z}=n_{\mathrm{i}} q \frac{\partial|V|}{\partial z}-k T_{\mathrm{i}} \frac{\partial n_{\mathrm{i}}}{\partial z}- & -n_{\mathrm{i}} m_{\mathrm{i}} \nu_{i \mathrm{~N}} v_{\mathrm{i}} .
\end{aligned}
$$

Le cas de la chute libre est celui où les deux derniers termes du deuxième membre sont négligeables : le terme collisionnel, par définition même de la chute libre, le terme de diffusion parce qu'il met en jeu l'énergie thermique ionique, faible devant l'énergie effective des ions dans la gaine. On vérifie en effet a posteriori que l'identité

$$
\frac{1}{n_{\mathrm{i}}}\left|\frac{\partial n_{\mathrm{i}}}{\partial z}\right|=\frac{1}{2}\left|\frac{1}{V} \frac{\partial V}{\partial z}\right|
$$

confrontée à l'inégalité

$$
|V|>k T_{\mathrm{e}} / q
$$

entraîne bien

$$
k T_{\mathrm{i}}\left|\frac{\partial n_{\mathrm{i}}}{\partial z}\right| \ll n_{\mathrm{i}} q\left|\frac{\partial V}{\partial z}\right|
$$

en tout point de la gaine.

En revanche, dans le cas collisionnel, la force de friction tend à compenser la force due au champ, ce qui a pour effet de rendre négligeable le premier membre de l'équation (32). Comme précédemment, le terme de diffusion est négligeable, et ce, d'autant plus que le champ est élevé. De fait, même à la lisière de gaine, cette approximation est raisonnable. En effet, en définissant la lisière, comme au paragraphe 3.1, par l'égalité simultanée des densités électronique et ionique et de leur dérivée spatiale (relations (11) et (12)), l'équation (32) s'écrit encore en ce point

$$
v_{\mathrm{i}} \simeq \mu_{\mathrm{i}} \frac{\partial|V|}{\partial z}-\frac{D_{\mathrm{i}}}{n_{\mathrm{e}}} \frac{\partial n_{\mathrm{e}}}{\partial z}
$$

soit, d'après la relation (23)

$$
v_{\mathrm{i}} \simeq \mu_{\mathrm{i}} \frac{\partial|V|}{\partial z}\left(1+\frac{T_{\mathrm{i}}}{T_{\mathrm{e}}}\right) \simeq \mu_{\mathrm{i}} \frac{\partial|V|}{\partial z} .
$$

La validité du modèle de chute libre est conditionnée par l'inégalité

$$
l>z-z_{\mathrm{s}}
$$

où $l$ est de l'ordre de grandeur des libres parcours moyens électronique et ionique. Elle entraîne l'absence d'ionisation dans la gaine et, par suite, la conservation du courant ionique (Eq. (8)). Le modèle collisionnel sans ionisation implique que soit 
vérifiée la double condition

$$
l_{\mathrm{I}}>z_{w}-z_{\mathrm{s}}>l
$$

où $l_{I}$ est le parcours électronique d'ionisation. En cas d'ionisation dans la gaine, il conviendrait de prendre en compte l'émission secondaire à la paroi et l'avalanche électronique. A ces effets, correspond un courant de paroi

$$
I_{\mathrm{i}}\left(z_{w}\right)=\frac{I_{\mathrm{i}}(z)}{1-\gamma_{\mathrm{T}}\left[\exp \alpha_{\mathrm{T}}\left(z_{w}-z\right)-1\right]}
$$

où $\alpha_{T}$ et $\gamma_{T}$ sont respectivement le $1^{\text {er }}$ et le $2^{\mathrm{e}}$ coefficient de Townsend. Ce courant est dominant par rapport au courant $I_{\mathrm{i}}\left(z_{\mathrm{s}}\right)$ injecté en lisière de gaine lorsque

$$
1-\gamma_{\mathrm{T}}\left[\exp \alpha_{\mathrm{T}}\left(z_{w}-z_{\mathrm{s}}\right)-1\right]=0 .
$$

Cette relation correspond à une troisième situation dans laquelle la gaine se comporte comme une gaine cathodique de décharge luminescente normale, d'épaisseur

$$
z_{w}-z_{\mathrm{s}}=\frac{1}{\alpha_{\mathrm{T}}} \log \frac{\dot{\gamma}_{\mathrm{T}}+1}{\gamma_{\mathrm{T}}} .
$$

Pour un gaz donné et des caractéristiques fixées des électrons du plasma, la valeur de la pression, $p$, permet de déterminer le modèle de gaine approprié. Dans le cas de l'Argon, et en prenant comme paramètre commun le courant ionique d'entrée de gaine et la chute de tension totale, W. B. Pennebaker [14] a tracé les courbes donnant en fonction de $p$ l'épaisseur des gaines pour les trois modèles cités plus haut. Les comparant à un calcul sans approximation, il fait ainsi apparaitre les domaines de validité correspondants et notamment, pour ce qui nous concerne, les limites des modèles développés aux paragraphes 3.1 et 3.2 (Fig. 1).

3.4 TEMPS DE MISE EN ÉQUILIBRE DE LA GAINE IONIQUE STATIONNAIRE. - $\mathrm{La}$ gaine est la «réponse » du plasma au potentiel de paroi. Une telle réponse est établie par la mise en mouvement des charges avec un temps de réaction égal au temps de transit des espèces les plus lourdes, c'est-à-dire des ions, au travers de la gaine. Ce temps est

$$
\tau=\int_{0}^{z_{w}-z_{\mathrm{s}}} \mathrm{d}\left(z-z_{\mathrm{s}}\right) / v_{\mathrm{i}}(z) .
$$

En utilisant les expressions en fonction du potentiel local, $V$, de $v_{\mathrm{i}}$ et de $\left(z-z_{\mathrm{s}}\right)$ calculé dans la limite de Child Langmuir, on obtient successivement

$$
\tau_{\mathrm{nc}} \sim 2\left(q\left|V_{w}\right| / k T_{\mathrm{e}}\right)^{1 / 4} / \omega_{\mathrm{pi}}\left(z_{\mathrm{s}}\right)
$$

pour la gaine non collisionnelle (nc) et

$$
\begin{aligned}
\tau_{\mathrm{c}} \sim 20\left(q\left|V_{w}\right| / k T_{\mathrm{e}}\right)^{1 / 3}\left(T_{\mathrm{e}} / T_{\mathrm{i}}\right)^{1 / 3} \times \\
\times\left[\nu_{\mathrm{iN}}\left(z_{w}\right) / \omega_{\mathrm{pi}}\left(z_{\mathrm{s}}\right)\right]^{1 / 3} / \omega_{\mathrm{pi}}\left(z_{\mathrm{s}}\right)
\end{aligned}
$$

pour les deux cas de gaine collisionnelle (c). $\nu_{\mathrm{iN}}\left(z_{w}\right)$ est la fréquence de collision calculée sur la paroi et $\omega_{\mathrm{pi}}\left(z_{\mathrm{s}}\right)$ est la pulsation plasma ionique à la lisière de gaine.

Dans le cas non collisionnel, $\tau_{\text {nc }}$ reste voisin de $2 \pi / \omega_{\mathrm{pi}}$ l'effet du potentiel de paroi étant très atténué par l'exposant 1/4. En revanche, les cas

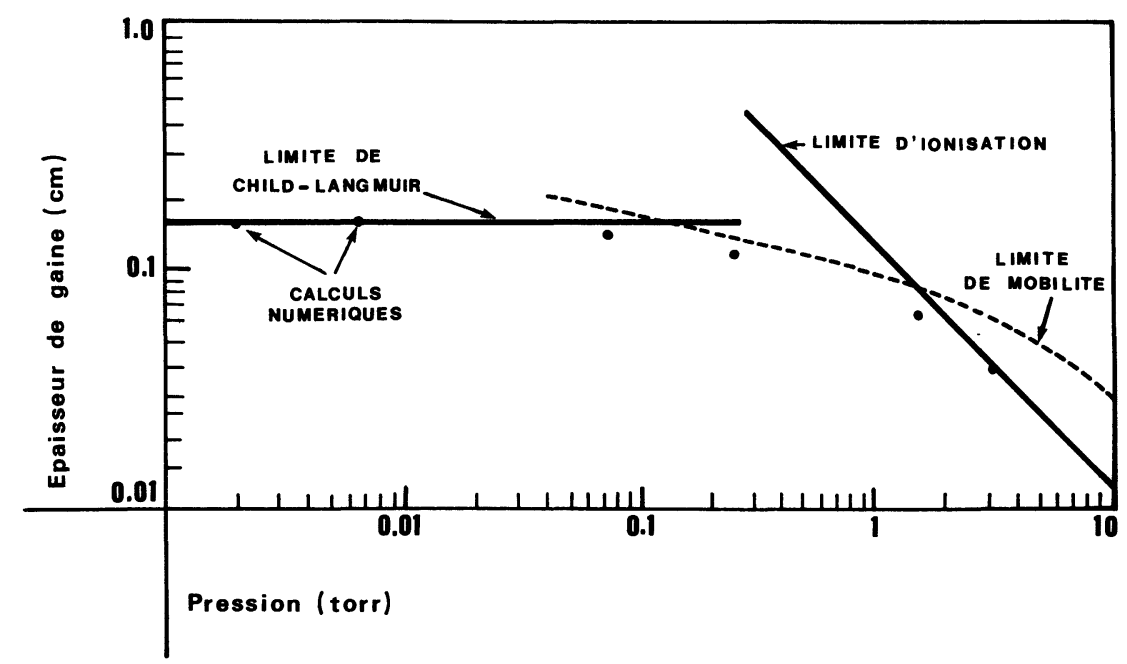

Fig. 1. - Comparaison des calculs numériques avec les différentes approximations de gaine. Les paramètres utilisés sont les suivants : Argon; Tension de gaine $=200 \mathrm{~V}$; densité de courant ionique à l'entrée de gaine $=10^{-3} \mathrm{~A} / \mathrm{cm}^{2}$; coefficient de Townsend : $\alpha / p=140$ (Torr.cm $)^{-1}, \gamma=0,2$. D'après [14].

[Comparison of numerical calculations with various sheath approximations. Operating parameters : Argon ; sheath voltage $=200 \mathrm{~V}$; ion current density entering the sheath $=10^{-3} \mathrm{~A} / \mathrm{cm}^{2} ;$ Townsend coefficients : $\alpha / p=140$ (Torr.cm $)^{-1}, \gamma=0.2$. From [14].] 
collisionnels, pour lesquels les ions dans la gaine sont thermalisés, ont une échelle de temps plus grande, à valeur de $n_{\mathrm{s}}$ fixée, essentiellement augmentée par le facteur

$$
\left(T_{\mathrm{e}} / T_{\mathrm{i}}\right)^{1 / 3}\left[\nu_{\mathrm{iN}}\left(z_{w}\right) / \omega_{\mathrm{pi}}\left(z_{\mathrm{s}}\right)\right]^{1 / 3} .
$$

3.5 GAINE QUASI STATIQUE. - Si l'on revient au cas général où le potentiel de paroi, donné par la relation (1), varie dans le temps, une première situation limite est celle pour laquelle $\omega \tau \ll 2 \pi$. C'est la limite « quasi stationnaire » où la gaine peut être considérée à chaque instant comme étant à l'équilibre statique vis-à-vis du potentiel. Alors le courant de conduction électronique est donné en fonction du potentiel instantané par les expressions établies dans le cas stationnaire : relation (6) pour la gaine sans collisions, relation (31) pour la gaine collisionnelle. Le courant $I_{\mathrm{i}}$, indépendant de $V$, garde une valeur constante. A ces courants de conduction doivent être ajoutés maintenant le courant de déplacement défini par la relation (3). En appliquant cette relation aux équations décrivant l'évolution spatiale du potentiel en gaine épaisse (relations de Child Langmuir successivement en chute libre (18) et limitées par la mobilité (27) et (28), on obtient :

$$
\begin{array}{r}
\left(I_{\mathrm{D}}\right)_{\mathrm{nc}}=\frac{\varepsilon_{0} A}{2}\left(\sqrt{\frac{m_{\mathrm{i}}}{2 q}} \frac{I_{\mathrm{i}}}{A \varepsilon_{0}}\right)^{1 / 2}\left|V_{w}\right|^{1 / 4} \times \\
\times\left(\frac{1}{\left|V_{w}\right|} \frac{\mathrm{d}\left|V_{w}\right|}{\mathrm{d} t}\right) \\
\left(I_{\mathrm{D}}\right)_{\mathrm{c}}=\varepsilon_{0} A \xi\left(\frac{I_{\mathrm{i}}}{A \mu_{\mathrm{i}}\left(z_{w}\right) \varepsilon_{0}}\right)^{1 / 3}\left|V_{w}\right|^{1 / 3} \times \\
\times\left(\frac{1}{\left|V_{w}\right|} \frac{\mathrm{d}\left|V_{w}\right|}{\mathrm{d} t}\right)
\end{array}
$$

avec $\xi=\frac{1}{2}\left(\frac{8}{9}\right)^{1 / 3}$ ou $\xi=\frac{2}{5}\left(\frac{3}{2}\right)^{1 / 3}$ lorsque le paramètre $\beta$ est respectivement égal à 0 et à $1 / 2$.

En posant $\left(1 / V_{w}\right)\left(\mathrm{d} V_{w}\right) / \mathrm{d} t \sim \omega$ et en considérant que l'échelle des courants de conduction est le courant ionique, $I_{\mathrm{i}}$, donné, suivant le régime, par les équations (16) ou (30), on obtient, par comparaison aux expressions (44) et (45)

$$
\left|I_{\mathrm{D}}\right| /\left|I_{\mathrm{c}}\right| \sim \omega \tau \ll 2 \pi
$$

Cette valeur du rapport $\left|I_{\mathrm{D}}\right| /\left|I_{\mathrm{c}}\right|$, obtenue pour un ordre de grandeur typique de $\left|I_{\mathrm{c}}\right|$ n'est évidemment pas valable aux instants où le potentiel de paroi est proche du potentiel flottant (pour lequel $I_{\mathrm{c}}=0$ ). $\mathrm{Si}$ l'on excepte ce cas (l'effet en sera discuté au paragraphe 5.2.1), elle montre que le comportement capacitif d'une gaine en équilibre quasi stationnaire est négligeable.
3.6 GAINE À IONS « GELÉS ». - La limite $\omega \tau \gg 2 \pi$ correspond à une situation différente dans laquelle l'équilibre statique décrit au paragraphe 3.4 ne peut jamais être atteint. La variation des conditions de paroi est en effet trop rapide pour que les ions puissent la prendre en compte : ils sont comme «gelés » c'est-à-dire qu'ils ne perçoivent que la valeur moyenne dans le temps, $\bar{V}$, du potentiel local

$$
V=\bar{V}(z)+\tilde{V}(z, t)
$$

la densité ionique est donc constante dans le temps

$$
n_{\mathrm{i}}=\bar{n}_{\mathrm{i}}(z)
$$

Les électrons, quant à eux, suivent le potentiel instantané pourvu que $2 \pi / \omega$ soit grand devant leur propre temps de transit, ce que nous supposerons vérifié. On peut montrer en effet, que cette dernière condition est peu restrictive pour les décharges qui nous intéressent puisqu'elle revient à l'inégalité $\omega \ll \omega_{\text {pe }}$ (cf. par exemple la référence [15] pour le cas de la chute libre). La densité électronique est donc fluctuante, de la forme

$$
n_{\mathrm{e}}=\bar{n}_{\mathrm{e}}(z)+\tilde{n}_{\mathrm{e}}(z, t) .
$$

Ainsi que précédemment, la lisière de gaine est définie comme étant la limite de séparation des charges, mais en raison de la fluctuation du potentiel de paroi, on peut imaginer que cette limite, d'abscisse $z_{\mathrm{s}}$, varie dans le temps. Trois conditions doivent donc être satisfaites [15]

$$
\begin{aligned}
& n_{\mathrm{i}}-n_{\mathrm{e}}=0 \quad \text { en } \quad z=z_{\mathrm{s}} \\
& \frac{\partial}{\partial z}\left(n_{\mathrm{i}}-n_{\mathrm{e}}\right)=0 \text { en } z=z_{\mathrm{s}} \\
& \frac{\mathrm{d}}{\mathrm{d} t}\left(n_{\mathrm{i}}-n_{\mathrm{e}}\right)=0 \quad \text { en } \quad z=z_{\mathrm{s}} \text {. }
\end{aligned}
$$

Les deux premières à $t$ fixé sont identiques aux conditions (11) et (12). La troisième implique que les électrons et les ions de la lisière évoluent ensemble dans le temps. Compte tenu de la condition (54) et de la relation (51), elle s'écrit encore

$$
\frac{\partial}{\partial t}\left(\bar{n}_{\mathrm{i}}-n_{\mathrm{e}}\right)=0 \quad \text { en } \quad z=z_{\mathrm{s}}
$$

d'où l'on déduit que la densité électronique à la lisière de gaine est indépendante du temps. Ceci n'est possible que si cette lisière et la valeur de $n_{\mathrm{s}}$ sont elles-mêmes constantes :

$$
\partial z_{\mathrm{s}} / \partial t=\partial n_{\mathrm{s}} / \partial t=0 .
$$

En d'autres termes l'épaisseur de gaine est constante et sa lisière fixée, contrairement au cas quasi statique où elle se déplaçait, obéissant à chaque instant aux conditions de l'équilibre stationnaire. Il y a néanmoins dans cette gaine un mouvement fluc- 
tuant d'électrons qui doit équilibrer la fluctuation du potentiel de paroi, $\tilde{V}_{\mathrm{w}}$, via l'équation de Poisson. Les deux comportements extrêmes de la gaine créée par un potentiel fluctuant, comportement quasi statique, d'une part, et à ions gelés, d'autre part, sont illustrés sur la figure 2 dans un système de coordonnées, $Z=z-z_{\mathrm{s}}$ lié à l'entrée de gaine.

L'équation de Poisson peut être séparée en deux parties :

$$
\begin{aligned}
& \partial^{2}|\bar{V}| / \partial z^{2}=\left(q / \varepsilon_{0}\right)\left(\bar{n}_{\mathrm{i}}-\bar{n}_{\mathrm{e}}\right) \\
& \partial^{2}|\tilde{V}| / \partial z^{2}=-\left(q / \varepsilon_{0}\right) \tilde{n}_{\mathrm{e}}
\end{aligned}
$$

La première concerne le potentiel moyen $\bar{V}$. Dans l'hypothèse de la gaine épaisse où, excepté au voisinage de $z_{\mathrm{s}}$ on peut supposer $n_{\mathrm{e}} \ll n_{\mathrm{i}}$, elle conduit aux solutions de Child Langmuir calculées aux paragraphes 3.1 et 3.2. Les valeurs de $\bar{V}$ coïncident donc avec celles de $V$ données par les relations (18, 27 et 28 ).

Dans le cadre de cette même hypothèse de la gaine épaisse, l'équation (59) qui décrit l'évolution spatiale de la partie fluctuante du potentiel, peut être approximée par l'équation de Laplace $\left(\tilde{n}_{\mathrm{e}}=0\right)$. Sa résolution, avec la condition aux limites approchée

$$
\tilde{V} \simeq 0 \quad \text { en } \quad z=z_{\mathrm{s}}
$$

donne

$$
\tilde{V} \simeq \tilde{V}_{w}\left(z-z_{\mathrm{s}}\right) /\left(z_{w}-z_{\mathrm{s}}\right) .
$$

L'épaisseur de gaine, $z_{w}-z_{\mathrm{s}}$, étant déductible de $\left|\bar{V}_{w}\right|$, il est donc possible d'exprimer en fonction du seul potentiel de paroi le courant de déplacement défini par la relation (3). On obtient ainsi :

$$
\begin{aligned}
& \left(I_{\mathrm{D}}\right)_{\mathrm{nc}}=-\frac{3}{4} \varepsilon_{0} A\left(\sqrt{\frac{8 m_{\mathrm{i}}}{q}} \frac{I_{\mathrm{i}}}{A \varepsilon_{0}}\right)^{1 / 2} \frac{1}{\left|\bar{V}_{w}\right|^{3 / 4}} \frac{\partial \tilde{V}_{w}}{\partial t} \\
& \left(I_{\mathrm{D}}\right)_{\mathrm{c}}=-\frac{2}{3} \varepsilon_{0} A\left(\frac{3 I_{\mathrm{i}}}{A \varepsilon_{0} \mu_{\mathrm{i}}}\right)^{1 / 3} \frac{1}{\left|\bar{V}_{w}\right|^{2 / 3}} \frac{\partial \tilde{V}_{w}}{\partial t} \quad \text { pour } \beta=0 \\
& \left(I_{\mathrm{D}}\right)_{\mathrm{c}}=-\frac{3}{5} \varepsilon_{0} A\left(\frac{5 I_{\mathrm{i}}}{2 A \varepsilon_{0}} \frac{\sqrt{p}}{K}\right)^{2 / 5} \frac{1}{\left|\bar{V}_{w}\right|^{3 / 5}} \frac{\partial \tilde{V}_{w}}{\partial t} \quad \text { pour } \beta=0,5
\end{aligned}
$$

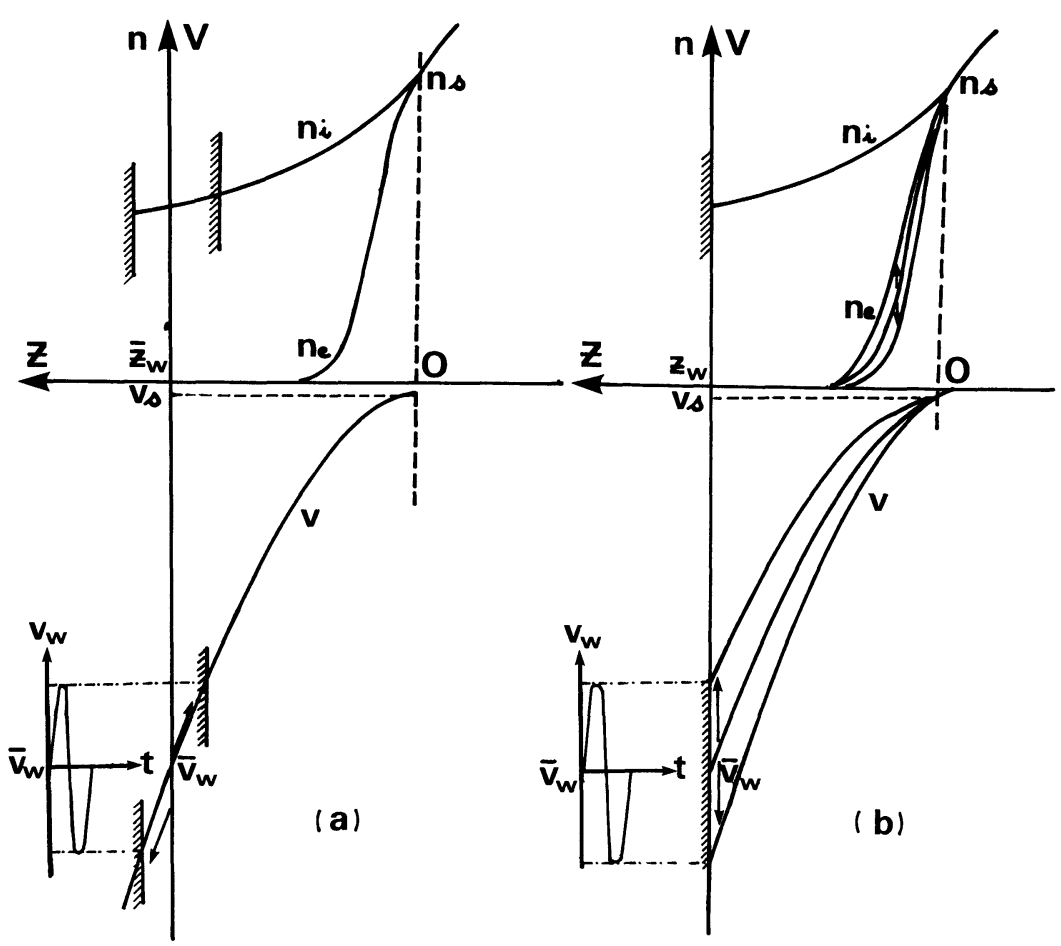

Fig. 2. - Comportements asymptotiques de la gaine en potentiel fluctuant dans un référentiel lié à l'entrée de gaine, $Z=z-z_{\mathrm{s}}$. a $: \omega \ll \omega_{\mathrm{pi}}$ (gaine quasi-statique) ; b : $\omega_{\mathrm{pi}} \ll \omega \ll \omega_{\mathrm{pe}}$ (gaine à ions gelés).

[Asymptotic behaviours of the sheath in a fluctuating potential, in a referential bound to the sheath edge, $Z=z-z_{\mathrm{s}} . \mathrm{a}: \omega \ll \omega_{\mathrm{pi}}$ (quasi-static sheath) $; \mathrm{b}: \omega_{\mathrm{pi}} \ll \omega \ll \omega_{\mathrm{pe}}$ (frozen ions sheath).] 
respectivement pour le cas de la chute libre et pour les deux cas collisionnels.

En prenant, ainsi qu'au paragraphe 3.4 , le courant $I_{\mathrm{i}}$ comme échelle du courant de conduction, et en posant $\mathrm{d} V_{w} / \mathrm{d} t \sim \omega V_{w}$ on trouve, dans les trois cas

$$
\left|I_{\mathrm{D}}\right| /\left|I_{\mathrm{c}}\right| \sim \omega \tau\left|\tilde{V}_{w}\right| /\left|\bar{V}_{w}\right|
$$

Pour un potentiel de paroi dont la composante fluctuante est du même ordre de grandeur que la valeur moyenne, et si l'on excepte dans l'alternance les temps où $V_{w}$ change de signe, le comportement de la gaine est essentiellement capacitif.

Dans les trois cas, la capacité équivalente de la gaine est constante et vaut :

$$
C=\varepsilon_{0} A /\left(z_{w}-z_{\mathrm{s}}\right)
$$

ce qui est évidemment, une conséquence de l'approximation $\tilde{n}_{\mathrm{e}} \simeq 0$ dans la gaine.

\section{Application des modèles de gaine aux décharges RF.}

4.1 PRINCIPES. - La connaissance des courants de conduction et de déplacement sur la paroi en fonction de son potentiel, mesuré par rapport au plasma, revient à celle de l'impédance de la gaine. Dans les cas $\omega \ll \omega_{\text {pi }}$ et $\omega \gg \omega_{\text {pi }}$ il est donc possible d'introduire les impédances des gaines des deux électrodes dans le schéma électrique complet de la décharge, et de déduire, au terme d'un calcul classique de circuit, l'évolution dans le temps des potentiels des électrodes. Dans le cadre de l'analyse développée aux paragraphes précédents, ces impédances dépendent à la fois du rapport $\omega / \omega_{\text {pi }}$ et du régime du mouvement ionique, correspondant à 6 possibilités. Un tel calcul a été fait à la référence [9] dans le cas de la chute libre pour $\omega \ll \omega_{\text {pi }}$. Ici, tenant compte des comportements dominants mis en évidence, nous procéderons à un traitement simplifié d'un plus grand nombre de cas, regroupés suivant le seul paramètre qu'est la fréquence :

1) lorsque $\omega \ll \omega_{\text {pi }}$, la gaine a un comportement quasi statique pour lequel le courant de conduction est dominant. Or celui-ci dépend seulement du potentiel de paroi et sa loi de variation prend la même forme, que la gaine soit collisionnelle ou non collisionnelle. On a vu en effet (relations (6) et (31)) que la composante électronique suit la même loi de décroissance exponentielle. Quant à la composante ionique, qui est indépendante de $V_{w}$, il est toujours possible de l'écrire

$$
I_{\mathrm{i}}=\left|I_{\mathrm{e}}\left(V_{\mathrm{f}}\right)\right|=\left|I_{\mathrm{ef}}\right|
$$

où $V_{\mathrm{f}}$ est le potentiel flottant, supposé connu. Le courant de conduction est donc

$$
I_{\mathrm{c}} \propto \exp \left(q V_{\mathrm{f}} / k T_{\mathrm{e}}\right)-\exp \left(q V_{w} / k T_{\mathrm{e}}\right),
$$

2) lorsque $\omega \gg \omega_{\mathrm{pi}}$, en revanche, la gaine a un comportement de type capacitif correspondant à un courant de déplacement dominant. Dans la limite de la gaine épaisse, on a vu (relations (62, 63 et 64)) que ce courant s'exprime sous la forme générale

$$
I_{\mathrm{D}}=A \frac{B_{\delta}}{\left|\bar{V}_{w}\right|^{\delta}} \frac{\partial \tilde{V}_{w}}{\partial t}
$$

où $B_{\delta}$ est un facteur multiplicatif et où l'exposant $\delta$ vaut respectivement $3 / 4,2 / 3$ et $3 / 5$ dans les cas de la chute libre, de la gaine collisionnelle à faible champ (mobilité ionique constante) et de la gaine collisionnelle à fort champ (mobilité ionique proportionnelle à $(\sqrt{p / E})$. Nous examinerons ces trois cas ensemble et nous ne les séparerons que pour les calculs numériques.

4.2 Configuration EXPÉRIMENTALE. - Soit une décharge diode RF. Elle est constituée de deux électrodes métalliques planes immergées dans le gaz de surfaces respectives $A_{1}$ et $A_{2}$, et qui se font face à une distance suffisante pour qu'il n'y ait pas recouvrement de leurs gaines électrostatiques. Les valeurs de $A_{1}$ et de $A_{2}$, sont, a priori, différentes dans le rapport

$$
\mathrm{A}_{1} / \mathrm{A}_{2}=\alpha .
$$

Les électrodes sont polarisées chacune à un potentiel $V_{j}(j=1,2)$ par une alimentation couplée par une capacité de blocage dont on suppose qu'elle ne modifie pas la forme sinusoïdale de la partie fluctuante de leur ddp.

On posera donc

$$
\tilde{V}_{1}-\tilde{V}_{2}=\tilde{V}_{\mathrm{RF}}=V_{\mathrm{RF}} \cos \omega t
$$

La masse du circuit électrique n'est pas précisée car elle est sans importance à ce stade de l'analyse.

D'un point de vue pratique cependant il conviendra d'en tenir compte pour évaluer la valeur effective de $\alpha$ : par exemple, dans le cas où une enceinte métallique à la masse confine le plasma, elle joue le rôle d'une électrode auxiliaire dont la surface doit être ajoutée à celle de l'électrode à la masse dans la relation $(70)$.

\subsection{ModÈle DE LA DÉCHARGE QUASI STATIQUE $\left(\omega \ll \omega_{\text {pi }}\right)$.}

4.3.1 Les équations. - Si les électrodes travaillaient comme deux sondes simples, elles obéiraient à des caractéristiques $I_{j}\left(V_{j}\right), j=1,2$, telles que pour une même valeur $V_{1}=V_{2}=V$ du potentiel,

$$
I_{1}(V) / I_{2}(V)=\alpha .
$$

Ceci implique en particulier que le potentiel flottant, $V_{\mathrm{f}}$ soit identique pour les deux électrodes. 
En présence de générateur externe qui fixe leur différence $V_{1}-V_{2}$, les potentiels des électrodes s'ajustent à chaque instant pour maintenir cette différence, tout en satisfaisant la loi de continuité des courants

$$
I_{1}\left(V_{1}\right)=-I_{2}\left(V_{2}\right) .
$$

La relation (73) traduit le fait que l'excès d'électrons reçu par l'électrode la plus positive circule dans le circuit extérieur jusqu'à l'électrode la plus négative, sur laquelle il est compensé par un égal excès d'ions venant du plasma. Elle concerne aussi bien la partie variable du courant $I_{j}$ que sa partie continue qui pourrait résulter d'une moyenne temporelle non nulle. La capacité externe impose donc la condition supplémentaire

$$
\int_{0}^{2 \pi / \omega} I_{1}\left(V_{1}\right) \mathrm{d} t=\int_{0}^{2 \pi / \omega} I_{2}\left(V_{2}\right) \mathrm{d} t=0 .
$$

La figure 3a montre aisément, que pour $\alpha \neq 1$, les relations (73) et (74) ne peuvent être simultanément vérifiées lorsque $V_{1}-V_{2}=V_{\mathrm{RF}} \cos \omega t$. On voit en effet que dans ces conditions, si la relation (73) est satisfaite à chaque instant, elle donne une moyenne temporelle du courant non nulle, correspondant à un excès d'ions. Comme dans le cas d'une sonde simple soumise à un potentiel alternatif [16], on s'attend à ce qu'il existe, pour compenser cet effet, un déplacement de la valeur moyenne des potentiels des électrodes, conduisant à la superposition d'un potentiel continu d'autopolarisation, $V_{\mathrm{DC}}$, au potentiel $V_{\mathrm{RF}}$. Une telle solution est illustrée par la figure $3 \mathrm{~b}$. On pose donc

$V_{1}-V_{2}=V_{\mathrm{DC}}+\tilde{V}_{\mathrm{RF}}=V_{10}-V_{20}+V_{\mathrm{RF}} \cos \omega t$

où $V_{\mathrm{j}_{0}}$ représente le potentiel de l'électrode $j$ à l'instant où $\tilde{V}_{\mathrm{RF}}=0$. Avec les nouvelles variables normalisées

$$
\eta_{\ell}=-q V_{\ell} / k T_{\mathrm{e}}
$$

où $l$ est un indice quelconque $\left(l=\mathrm{j}, \mathrm{j}_{0}, \mathrm{f}\right)$, et

$$
a=q V_{\mathrm{RF}} / k T_{\mathrm{e}} .
$$

Les relations (73, 74 et 75), compte tenu de l'expression (68) des courants $I_{\mathrm{j}}=I_{\mathrm{Cj}}$ et de leur valeur relative donnée par la relation (72), conduisent respectivement aux équations :

$$
\begin{gathered}
\alpha\left\{\exp \left[-\eta_{1}(t)\right]-\exp \left[-\eta_{\mathrm{f}}\right]\right\}= \\
=\exp \left[-\eta_{\mathrm{f}}\right]-\exp \left[-\eta_{2}(t)\right] \\
\int_{0}^{2 \pi / \omega}\left\{\exp \left[-\eta_{1}(t)\right]-\exp \left[-\eta_{\mathrm{f}}\right]\right\} \mathrm{d} t=0 \\
\eta_{1}-\eta_{2}=\eta_{10}-\eta_{20}-a \cos \omega t
\end{gathered}
$$

Ces trois relations sont valables à chaque instant $t$,
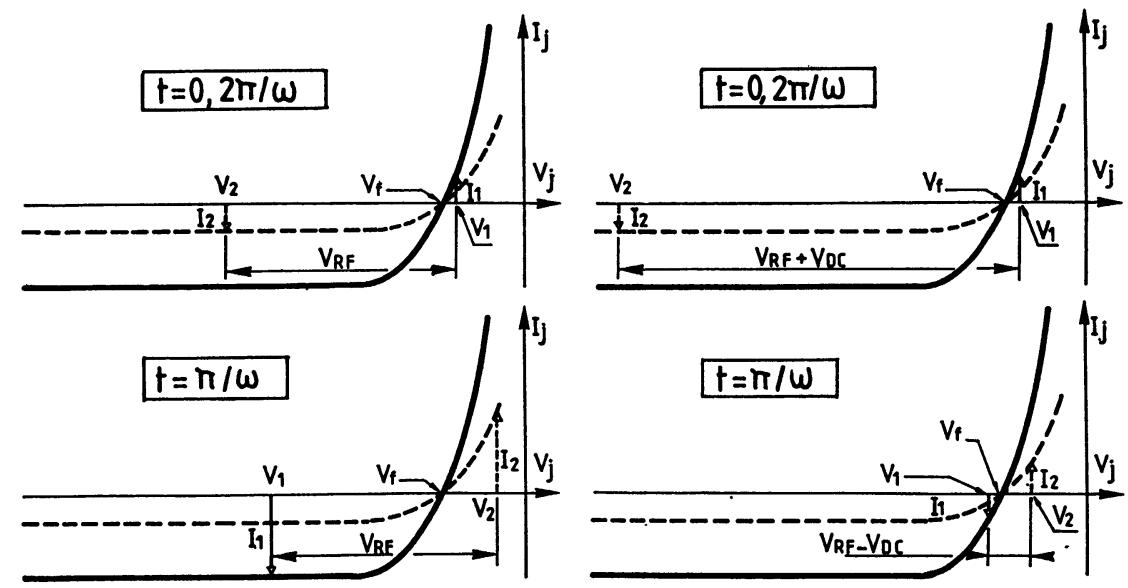

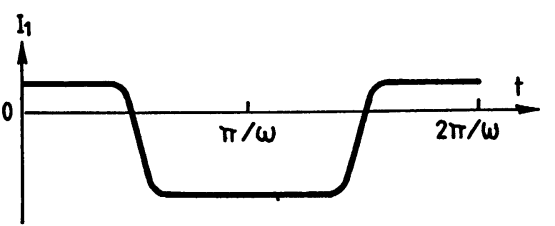

(a)

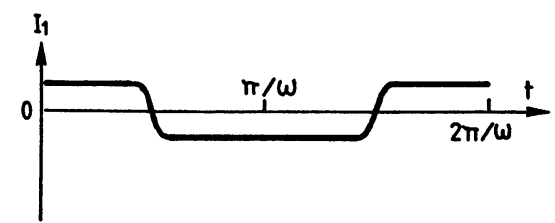

(b)

Fig. 3. - Points de fonctionnement, à différents instants, sur les caractéristiques de sonde, $I_{j}\left(V_{j}\right), j=1,2$, des électrodes d'une décharge asymétrique, et variations correspondantes du courant extérieur $I_{1}=-I_{2}\left(\omega \ll \omega_{\mathrm{pi}}\right)$. $a$ : sans tension d'autopolarisation; $b$ : avec tension d'autopolarisation.

[Working points, at different moments, on the probe characteristics, $I_{j}\left(V_{j}\right), j=1,2$, of the electrodes of an asymmetrical discharge, and related variations of the external current $I_{1}=-I_{2}\left(\omega \ll \omega_{\mathrm{pi}}\right)$.

$a$ : without self-bias voltage ; $b$ : with self-bias voltage.] 
en particulier la relation (78) lorsque $\cos \omega t=0$, ce qui donne

$$
\begin{aligned}
\alpha\left\{\exp \left[-\eta_{10}\right]-\exp \left[-\eta_{\mathrm{f}}\right]\right\}= & \\
= & \exp \left[-\eta_{\mathrm{f}}\right]-\exp \left[-\eta_{20}\right] .
\end{aligned}
$$

La résolution du système d'équations (78) à (81) permet de déterminer les quatre inconnues $\eta_{1}(t)$, $\eta_{2}(t), \eta_{10}$ et $\eta_{20}$ en fonction des paramètres $a$ et $\alpha$.

4.3.2 Evoluation temporelle des potentiels des électrodes. - Lorsque les deux électrodes ont une même surface $(\alpha=1)$, il existe une solution analytique pour toutes les valeurs de $a$

$\eta_{\mathrm{j}}-\eta_{\mathrm{f}}=(-1)^{\mathrm{j}} \frac{a \cos \omega t}{2}+\log \left\{\operatorname{ch} \frac{a \cos \omega t}{2}\right\}$.

Ces potentiels d'électrode varient donc de façon semblable avec un décalage temporel d'une demipériode et une valeur identique lorsque $\cos \omega t=0$ $\left(\eta_{10}=\eta_{20}=\eta_{\mathrm{f}}\right)$. L'absence de potentiel d'autopolarisation signifie que le courant moyen collecté par chaque électrode est nul. Ceci était, a priori, évident puisque le courant instantané collecté par l'électrode 1 est alors identique au courant extérieur d'une double sonde flottante symétrique, soit [12]:

$$
I_{1}=\left|I_{\text {ef }}\right| \text { th } \frac{a \cos \omega t}{2}
$$

$I_{1}$ représente la limite de la courbe correspondante de la figure 3a lorsque $\alpha \rightarrow 1$. Les variations de $V_{1}(t)-V_{\mathrm{f}}$ et de $V_{2}(t)-V_{\mathrm{f}}$ sont montrées sur la figure $4 \mathrm{a}$.
Lorsque les plaques sont dissymétriques $(\alpha>1)$, $\eta_{1}(t)$ et $\eta_{2}(t)$ peuvent encore être exprimés analytiquement sous la forme

$$
\begin{aligned}
& \eta_{\mathrm{j}}-\eta_{\mathrm{f}}=(-1)^{\mathrm{j}} \frac{a \cos \omega t+\eta_{20}-\eta_{10}}{2}+ \\
& +\log \left[\frac{2 \sqrt{2}}{1+\alpha} \operatorname{ch} \frac{a \cos \omega t+\log \alpha+\eta_{20}-\eta_{10}}{2}\right] .
\end{aligned}
$$

En revanche, les calculs de $\boldsymbol{\eta}_{10}$ et $\boldsymbol{\eta}_{20}$ doivent être effectués numériquement. Pour des valeurs élevées du paramètre $a(a>20)$, les résultats mettent en évidence des lois simples de variation de $\eta_{\mathrm{j}_{0}}$ avec $a$, qui sont explicitées dans les trois dernières lignes du tableau I. Ces lois font intervenir un facteur $\zeta$ qui apparait, entre autres, comme étant la pente de la variation avec $a$ du potentiel réduit d'autopolarisation, $\eta_{20}-\eta_{10}$. Les deux lignes immédiatement supérieures du tableau présentent les lois qui s'en déduisent pour la variation des valeurs maximales $\eta_{\mathrm{jM}}$, de $\eta_{\mathrm{j}}$. Le facteur $\zeta$ dépend lui-même du paramètre $\alpha$, comme le montre la figure $5:$ il croît de 0 à 1 lorsque $\alpha$ croît de 1 à l'infini.

Lorsque $\alpha$ est soit voisin de 1 , soit grand devant 1 , des solutions analytiques approchées sont possibles pour $\eta_{10}$ et $\eta_{20}$, correspondant à des expressions pour $\zeta$ et pour la valeur moyenne $\bar{\eta}_{\mathrm{j}}$ de $\eta_{\mathrm{j}}$, qui sont portées dans les trois premières lignes du tableau. Les gammes de validité correspondantes pour $\alpha$ ont été estimées en comparant les lois approchées, $\zeta(\alpha)$, avec la solution numérique (cf. Fig. 5).

L'examen du tableau I montre donc qu'il existe toujours un potentiel d'autopolarisation, $\eta_{20}-\eta_{10}$
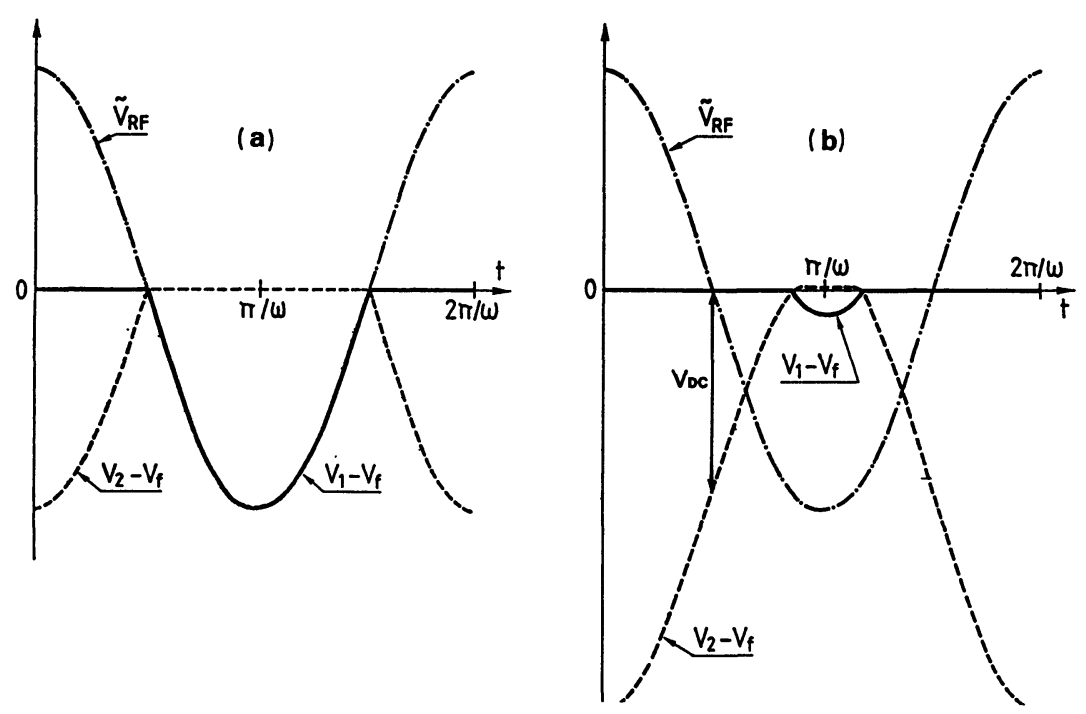

Fig. 4. - Variation temporelle des potentiels d'électrode : cas $\omega \ll \omega_{\mathrm{pi}}$ avec $V_{\mathrm{RF}}=100 \mathrm{kT} / \mathrm{e}$. a : décharge symétrique $(\alpha=1) ;$ b : décharge asymétrique $(\alpha=7)$.

[Time variation of the plates potentials : case $\omega \ll \omega_{\mathrm{pi}}$ with $V_{\mathrm{RF}}=100 k T_{\mathrm{e}} / q$. a : symmetrical discharge $(\alpha=1) ; \mathrm{b}$ : asymmetrical discharge $(\alpha=7)$.] 
Tableau I. - Lois de variation analytiques pour les valeurs élevées du potentiel RF réduit, a, en fonction du rapport des surfaces d'électrode, $\alpha$, et du facteur $\zeta$ de la figure 5. (Les trois premières lignes sont des approximations valables pour les valeurs extrêmes de $\alpha$.)

[Asymptotical variation laws for high values of the normalized RF voltage, $a$, versus the electrodes areas ratio, $\alpha$, and the $\zeta$ factor appearing in figure 5. (The three first lines are approximations valid for extreme values of $\alpha$.$) ]$

\begin{tabular}{|l|l|l|}
\cline { 2 - 3 } \multicolumn{1}{c|}{} & \multicolumn{2}{|c|}{$a>20$} \\
\cline { 2 - 3 } \multicolumn{1}{c|}{} & $1<\alpha<2$ & $7<\alpha$ \\
\hline$\zeta$ & $\pi\left(\frac{\alpha}{1+\alpha}-\frac{1}{2}\right)$ & $\left(1-\frac{\pi^{2}}{2 \alpha^{2}}\right)$ \\
\hline $\bar{\eta}_{1}-\eta_{\mathrm{f}}$ & $\frac{a}{\pi}\left[1-\frac{\pi^{2}}{2} \zeta(1-\zeta)\right]+\log \frac{\alpha}{1+\alpha}$ & $\frac{\pi^{2}}{2 \alpha^{3}} a+\log \frac{\alpha}{1+\alpha}$ \\
\hline $\bar{\eta}_{2}-\eta_{\mathrm{f}}$ & $\frac{a}{\pi}\left[1-\frac{\pi^{2}}{2} \zeta(3-\zeta)\right]+\log \frac{\alpha^{2}}{1+\alpha}$ & $a \zeta-\log (1+\alpha)$ \\
\hline$\eta_{1 \mathrm{M}}-\eta_{\mathrm{f}}$ & $(1-\zeta) a+\log \frac{\alpha}{1+\alpha}$ \\
\hline$\eta_{2 \mathrm{M}}-\eta_{\mathrm{f}}$ & & $(1+\zeta) a-\log (1+\alpha)$ \\
\hline$\eta_{\mathrm{f}}-\eta_{10}$ & \multicolumn{2}{|c|}{$\frac{(1+\alpha) \exp (\zeta a)}{\alpha[1+\exp (\zeta a)]}$} \\
\hline$\eta_{\mathrm{f}}-\eta_{20}$ & \multicolumn{2}{c|}{\begin{tabular}{c}
$1+\alpha$ \\
\hline$\eta_{20}-\eta_{10}$
\end{tabular}} \\
\hline
\end{tabular}

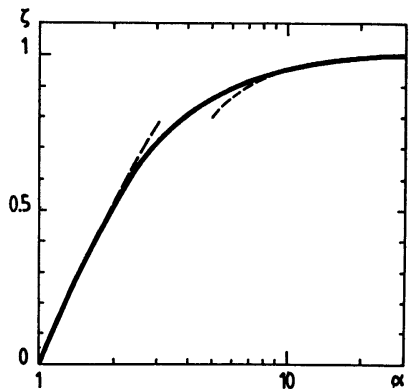

Fig. 5. - Variation, pour $V_{\mathrm{RF}}$ grand et $\omega \ll \omega_{\mathrm{pi}}$, de la pente de la tension d'autopolarisation avec le rapport des surfaces d'électrode : lois approchée (tiretés) et résultats numériques (trait plein).

[Variation, for large $V_{\mathrm{RF}}$ and $\omega \ll \omega_{\mathrm{pi}}$, of the self-bias voltage slope versus ratio of electrodes surface areas : approximate law (dashed line) and numerical results (solid line).]

pour $\alpha \neq 1$. Dans ce potentiel, la contribution de la petite électrode est la plus importante, tendant à n'être que la seule lorsque $\alpha \rightarrow \infty$. Cette dernière situation est celle où la grande électrode reste polarisée au potentiel flottant $\left(\eta_{10} \simeq \eta_{1}(t) \simeq \eta_{\mathrm{f}}\right)$, la petite électrode se comportant alors comme une sonde simple. On retrouve bien dans ce cas une valeur $\eta_{20} \simeq a$, limite de l'effet de redressement étudié en [16] lorsque $a$ est grand. A fins de comparaison avec le cas symétrique, la figure $4 \mathrm{~b}$ présente pour une valeur de $\alpha$ grande mais non « infinie » $(\alpha=7)$, les variations temporelles des potentiels d'électrode, repérés par rapport au potentiel flottant.

4.3.3 Rôle de la capacité de blocage. - Dans le cas où la tension d'excitation est appliquée directement, sans capacité de blocage il n'y a de courant moyen collecté dans le circuit extérieur que si $\alpha \neq 1$. Les résultats présentés pour les électrodes symétriques restent donc inchangés. En revanche, pour des électrodes dissymétriques, la suppression de la condition (74) revient à annuler le potentiel d'autopolarisation $V_{10}-V_{20}$ dans la relation (75). Les solutions $\eta_{\mathrm{j}}$ données par la relation (84) prennent alors la forme

$$
\begin{aligned}
\eta_{\mathrm{j}}-\eta_{\mathrm{f}} & =(-1)^{\mathrm{j}} \frac{a \cos \omega t}{2}+ \\
+ & \log \left[\frac{2 \sqrt{\alpha}}{1+\alpha} \operatorname{ch} \frac{a \cos \omega t+\log \alpha}{2}\right] .
\end{aligned}
$$


Il est important de remarquer que la dissymétrie entre la polarisation de la grande et celle de la petite électrode n'intervient plus ici que dans un terme logarithmique, négligeable si l'amplitude $a$ est grande. Dans cette limite, les variations de $V_{1}(t)$ et $V_{2}(t)$ restent données par les courbes de la figure $4 a$.

4.3.4 Modulation du potentiel plasma. - Les potentiels $V_{\mathrm{j}}$ sont repérés par rapport au potentiel plasma : $V_{\mathrm{j}}=\Phi_{\mathrm{j}}-\Phi_{\mathrm{p}}$.

Si le potentiel absolu, $\Phi_{\mathrm{j}}$, de l'une des électrodes est fixé en étant par exemple mis à la masse, le potentiel plasma est donc lui-même modulé. On le déduit aisément des lois étudiées en 4.3.2 (et 4.3.3 s'il n'y a pas de capacité de liaison) par les relations

$$
\begin{array}{lll}
\Phi_{\mathrm{p}}=\eta_{1} k T_{\mathrm{e}} / q & \text { si } & \Phi_{1}=0 \\
\Phi_{\mathrm{p}}=\eta_{2} k T_{\mathrm{e}} / q & \text { si } & \Phi_{2}=0 .
\end{array}
$$

La figure 6 présente des exemples de courbes
$\Phi_{\mathrm{p}}(t)$ obtenues avec ou sans capacité de liaison et pour différentes configurations expérimentales.

4.3.5 Dispersion de l'énergie des ions. - Les ions qui traversent la gaine en un temps inférieur à sa période de modulation ont sur l'électrode une vitesse liée à la valeur instantanée du potentiel d'électrode. Dans le cas non collisionnel, par exemple, leur énergie cinétique, déduite de la relation (7) est

$$
E_{\mathrm{i}}=q\left|V_{\mathrm{j}}\right|(t) .
$$

Le flux correspondant, qui est indépendant du potentiel et donc du temps, s'écrit

$$
\mathrm{d} N_{\mathrm{i}} / \mathrm{d} t=I_{\mathrm{i}} / q
$$

ce qui conduit à une distribution en énergie

$$
g\left(E_{\mathrm{i}}\right)=\mathrm{d} N_{\mathrm{i}} / \mathrm{d} E_{\mathrm{i}}=I_{\mathrm{i}} /\left\{q^{2} \mathrm{~d}\left|V_{\mathrm{j}}\right| / \mathrm{d} t\right\} .
$$

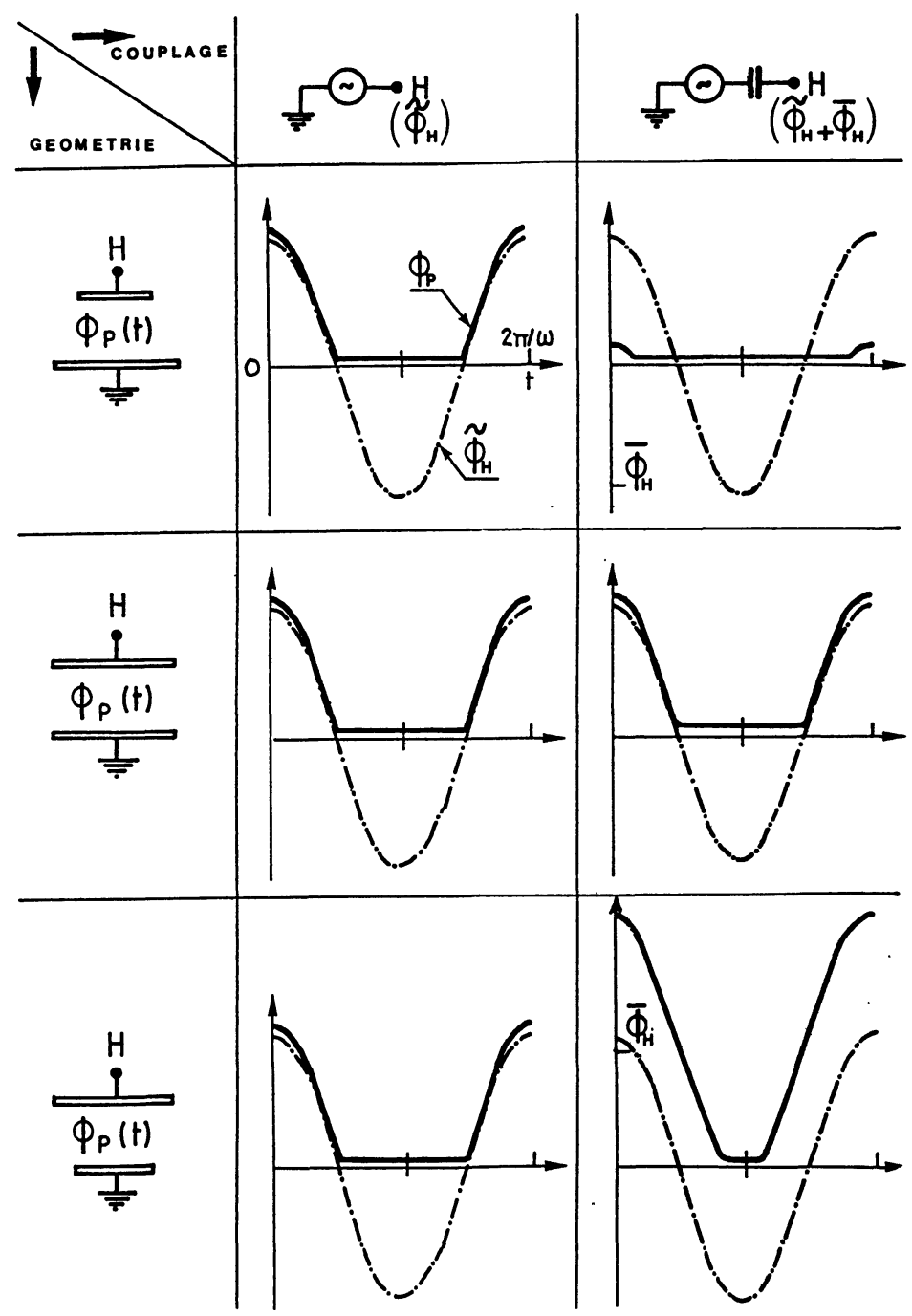

Fig. 6. - Variation temporelle du potentiel plasma (traits pleins) pour trois configurations géométriques en l'absence et en présence de capacité de blocage sur l'électrode d'excitation, $\mathrm{H}:$ cas $\omega \ll \omega_{\mathrm{pi}}$ avec $\alpha=7, V_{\mathrm{RF}}=100 k T_{\mathrm{e}} / q$, $\left|V_{\mathrm{f}}\right|=5 k T_{\mathrm{c}} / q$.

[Temporal variation of the plasma potential (solid line) for three geometrical configurations with and without a blocking capacitor on the high voltage side, $\mathrm{H}:$ case $\omega \ll \omega_{\mathrm{pi}}$ and $\alpha=7, V_{\mathrm{RF}}=100 k T_{\mathrm{e}} / q,\left|V_{\mathrm{f}}\right|=5 k T_{\mathrm{e}} / q$.] 
La loi $V_{\mathrm{j}}(t)$ étant connue par l'étude du paragraphe 4.3.2, cette distribution peut être calculée. En particulier, si l'on s'intéresse aux ions collectés par la grande électrode, on obtient, à partir de la relation (84)

$\eta_{1}-\eta_{\mathrm{f}} \simeq-a \cos \omega t-\zeta a+\log [(1+\alpha) / \alpha]$

lorsque $\pi(1-1 / \alpha)<\omega t<\pi$ d'où, en revenant aux variables non normalisées :

$$
\frac{\mathrm{d}\left|V_{1}\right|}{\mathrm{d} t} \simeq V_{\mathrm{RF}} \omega \sqrt{2 \frac{\left|V_{1}\right|_{\mathrm{M}}}{V_{\mathrm{RF}}}} \sqrt{1-\frac{\left|V_{1}\right|}{\left|V_{1}\right|_{\mathrm{M}}}}
$$

pour $\left|V_{1}\right| \geq\left|V_{\mathrm{f}}\right|$. On en déduit

$$
g\left(E_{\mathrm{i}}\right) \propto \frac{1}{\sqrt{1-E_{\mathrm{i}} / E_{\mathrm{iM}}}}
$$

où $E_{\mathrm{iM}}$ représente l'énergie maximum des ions collectés correspondant au maximum de potentiel attractif de gaine $\left|V_{1}\right|_{M}$.

\subsection{MOdèle DE LA DÉCHARge A IONS GelÉs $\omega \gg \omega_{\mathrm{pi}}$.}

4.4.1 Equations. - Conformément au modèle de gaine développé au paragraphe 3.6 , il convient de distinguer dans le potentiel de l'électrode $\mathrm{j}$ la partie constante dans le temps et la partie fluctuante, à moyenne nulle. Avec les mêmes notations que précédemment, le potentiel réduit prend la forme

$$
\eta_{\mathrm{j}}=\bar{\eta}_{\mathrm{j}}+\tilde{\eta}_{\mathrm{j}}(t)
$$

Il lui correspond un courant collecté, somme du courant de conduction et du courant de déplacement, qui s'écrit

$I_{\mathrm{j}}=A_{\mathrm{j}}\left\{D_{\delta}\left[\exp \left(-\eta_{\mathrm{f}}\right)-\exp \left(-\eta_{\mathrm{j}}\right)\right]+\mathrm{F}_{\delta} \frac{1}{\bar{\eta}_{\mathrm{j}}^{\delta}} \frac{\partial \tilde{\eta}_{\mathrm{j}}}{\partial t}\right\}$

où les constantes $D_{\delta}$ et $F_{\delta}$ sont aisément déductibles des relations $(6,31,62,63,64)$.

Par analogie avec le traitement quasi stationnaire nous supposerons que le générateur donne la ddp entre électrodes :

$$
\eta_{1}-\eta_{2}=\eta_{10}-\eta_{20}-a \cos \omega t
$$

où $\eta_{\mathrm{j}_{0}}$ est défini par la condition

$$
\eta_{\mathrm{j}}=\eta_{\mathrm{j}_{0}} \text { quand } \cos \omega t=0 .
$$

La loi de continuité des courants et la condition de nullité de la valeur moyenne du courant $I_{\mathrm{j}}$ conduisent aux relations

$$
\begin{aligned}
& \alpha\left\{D_{\delta}\left[\exp \left(-\eta_{\mathrm{f}}\right)-\exp \left(-\eta_{1}\right)\right]+F_{\delta} \frac{1}{\bar{\eta}_{1}^{\delta}} \frac{\partial \tilde{\eta}_{1}}{\partial t}\right\}= \\
& \left.=-\left\{D_{\delta}\left[\exp \left(-\eta_{\mathrm{f}}\right)-\exp \left(-\eta_{2}\right)\right]+F_{\delta} \frac{1}{\bar{\eta}_{2}^{\delta}} \frac{\partial \tilde{\eta}_{2}}{\partial t}\right\}\right\}
\end{aligned}
$$

$$
\int_{0}^{2 \pi / \omega}\left[\exp \left(-\eta_{\mathrm{f}}\right)-\exp \left(-\eta_{1}\right)\right] \mathrm{d} t=0 \text {. }
$$

4.4.2 Evolution temporelle des potentiels d'électrode. - Les relations $(96,97,98,99)$ permettent en principe de déterminer les quatre inconnues $\eta_{1}(t) \eta_{2}(t), \eta_{10}$ et $\eta_{20}$. Dans un but de simplification, conformément au principe énoncé au paragraphe 4.1, nous les résoudrons en supposant que $I_{\mathrm{Dj}} \gg I_{\mathrm{Cj}}$ dans la relation (98). Celle-ci se réduit alors à l'équation

$$
\frac{\partial}{\partial t}\left(\gamma_{\delta} \eta_{1}+\eta_{2}\right)=0
$$

où

$$
\gamma_{\delta}=\alpha\left(\bar{\eta}_{2} / \bar{\eta}_{1}\right)^{\delta} .
$$

La résolution du système simplifié pour les valeurs élevées de la tension d'excitation, donne

$$
\begin{gathered}
\eta_{1}-\eta_{\mathrm{f}} \simeq\left[a /\left(\gamma_{\delta}+1\right)\right][1-\cos \omega t] \\
\text { si } a>\gamma_{\delta}+1 \\
\eta_{2}-\eta_{\mathrm{f}} \simeq\left[\gamma_{\delta} a /\left(\gamma_{\delta}+1\right)\right][1+\cos \omega t] \\
\text { si } \gamma_{\delta} a>\gamma_{\delta}+1 .
\end{gathered}
$$

On remarque que, contrairement au cas des basses fréquences

$$
\eta_{\mathrm{j}_{0}}=\bar{\eta}_{\mathrm{j}}
$$

avec

$$
\begin{aligned}
& \bar{\eta}_{1}-\eta_{\mathrm{f}}=a /\left(\gamma_{\delta}+1\right) \\
& \bar{\eta}_{2}-\eta_{\mathrm{f}}=\gamma_{\delta} a /\left(\gamma_{\delta}+1\right) .
\end{aligned}
$$

La prise en compte de ces deux dernières relations dans la relation (101) permet d'exprimer $\gamma_{\delta}$ en fonction de $\alpha$ au travers de l'équation

$$
\alpha=\gamma_{\delta}\left[\left(b+\gamma_{\delta}+1\right) /\left(\gamma_{\delta} b+\gamma_{\delta}+1\right)\right]^{\delta}
$$

avec

$$
b=a / \eta_{\mathrm{f}}=V_{\mathrm{RF}} /\left|V_{\mathrm{f}}\right| .
$$

Excepté pour le cas $\alpha=1$ où $\gamma_{\delta}=1$ quelle que soit la valeur de $b$, le coefficient $\gamma_{\delta}$ dépend à la fois du rapport des surfaces des électrodes et du potentiel d'excitation. Les courbes $\gamma_{\delta}(\alpha)$, calculées à partir de la relation (107) pour différentes valeurs du paramètre $b$, sont portées sur les figures $4 a, 7 b, 7 c$, respectivement pour les trois modèles de gaine envisagés. 

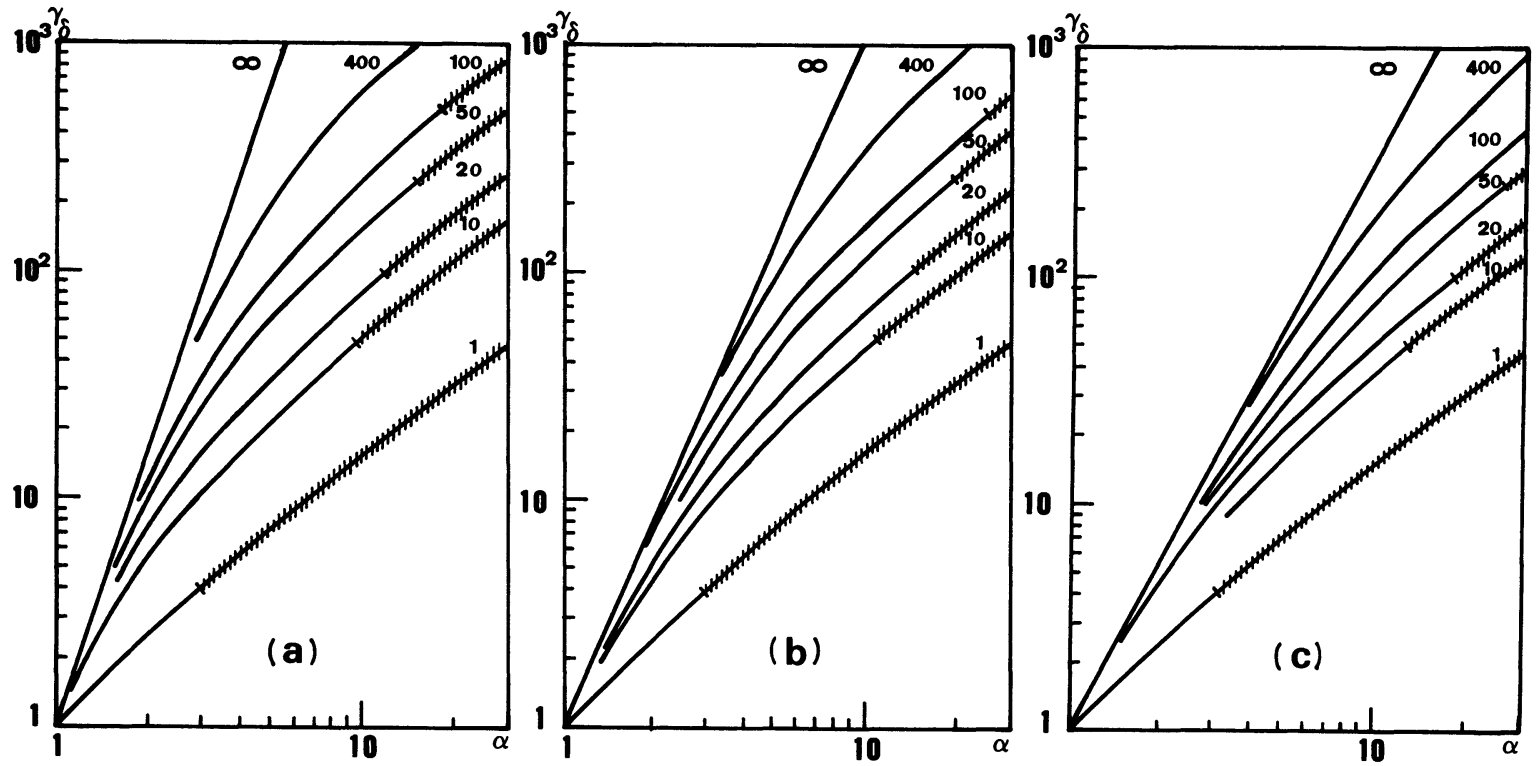

Fig. 7. - Variation du facteur $\gamma_{\delta}$ en fonction du rapport des surfaces d'électrode pour différentes valeurs du paramètre $V_{\mathrm{RF}} /\left|V_{\mathrm{f}}\right|: \mathrm{a}: \delta=3 / 4 ; \mathrm{b}: \delta=2 / 3 ; \mathrm{c}: \delta=3 / 5$.

[Variation of the factor $\gamma_{\delta}$ as a function of the ratio of electrodes surface areas for different values of the parameter $V_{\mathrm{RF}} / V_{\mathrm{f}}: \mathrm{a}: \delta=3 / 4 ; \mathrm{b}: \delta=2 / 3 ; \mathrm{c}: \delta=3 / 5$.]

Dans le cas de la chute libre $(\delta=3 / 4)$, on remarque que $\gamma \rightarrow \alpha^{4}$ lorsque $b \rightarrow \infty$. Une telle limite, obtenue quand $\eta_{\mathrm{f}} \rightarrow 0$, conduit, conformément à (101), à la relation $\bar{\eta}_{2} / \bar{\eta}_{1}=\alpha^{4}$, identique à la loi donnée aux références [1,2] et [5]. De même, la limite $b \rightarrow \infty$ correspond aux lois asymétriques $\eta_{2} / \eta_{1}=\alpha^{3}$ et $\eta_{2} / \eta_{1}=\alpha^{5 / 2}$, respectivement pour les cas $\delta=2 / 3$ et $\delta=3 / 5$. Lorsque $b=1$, les courbes $\gamma_{\delta}(\alpha)$ sont quasi linéaires et ne différent pour les trois valeurs de $\delta$ que par un coefficient multiplicatif qui reste du même ordre. L'influence de $\delta$, à $\alpha$ fixé, est d'autant plus grande que le paramètre $b$ est plus élevé. A titre d'exemple, on note que, pour $\alpha=7$ et $b=20$, les trois valeurs de $\gamma$ correspondant à $\delta=3 / 4,2 / 3$ et $3 / 5$ sont respectivement égales à 55,40 et 33 .

Sur les trois figures $7 a, 7 b$ et $7 c$, les hachures indiquent les régions dans lesquelles le paramètre a est trop petit pour que les relations (102) et (103) soient valables. Elles ont été tracées pour le cas $\eta_{\mathrm{f}}=5$.

4.4.3 Rôle de la capacité de blocage et modulation du potentiel plasma. - Comme cela était le cas au paragraphe 4.3.3, la suppression de la capacité de blocage a pour effet d'annuler la tension d'autopolarisation $\eta_{20}-\eta_{10}$ dans la relation (96) et de supprimer la condition d'un courant moyen nul, exprimée par la relation (99). La valeur moyenne des potentiels d'électrode est alors la même

$$
\bar{\eta}_{1}=\bar{\eta}_{2}=\bar{\eta}
$$

ce qui correspond, d'après la relation (101) à

$$
\gamma_{\delta}=\alpha
$$

quel que soit $\delta$ et donc quel que soit le modèle de gaine envisagé. Le potentiel $\eta$ qui contribue à la constante d'intégration de la relation de continuité simplifiée (100) doit être calculé en intégrant sur une période l'équation de continuité initiale (98). On obtient ainsi

$$
\bar{\eta}=\eta_{10}=\eta_{20} \simeq \eta_{\mathrm{f}}+\frac{\alpha a}{\alpha+1} \quad \text { lorsque } \quad \frac{\alpha a}{\alpha+1} \gg 1
$$

ce qui conduit aux nouvelles lois de variation

$$
\begin{aligned}
& \eta_{1}-\eta_{\mathrm{f}}=\frac{\alpha a}{\alpha+1}\left(1-\frac{1}{\alpha} \cos \omega t\right) \\
& \eta_{2}-\eta_{\mathrm{f}}=\frac{\alpha a}{\alpha+1}(1+\cos \omega t) .
\end{aligned}
$$

Comme précédemment au paragraphe 4.3.4 la fluctuation du potentiel absolu $\Phi_{\mathrm{p}}$ lorsque l'une des électrodes est à la masse peut être aisément déduite des relations (102) et (103) ou (111) et (112). Des exemples d'une telle fluctuation sont montrés sur la figure 8 avec les mêmes valeurs des paramètres $\alpha$, $V_{\mathrm{f}}$ et $V_{\mathrm{RF}}$ que pour la figure 6 .

\section{Discussion des résultats.}

\subsection{COMPARAISON A DES RÉSUlTATS EXPÉRIMEN- TAUX.}

5.1.1 La référence [6] présente des mesures effectuées sur une décharge RF plane asymétrique $(\alpha \simeq 6)$ à couplage capacitif dans l'argon à moyenne 


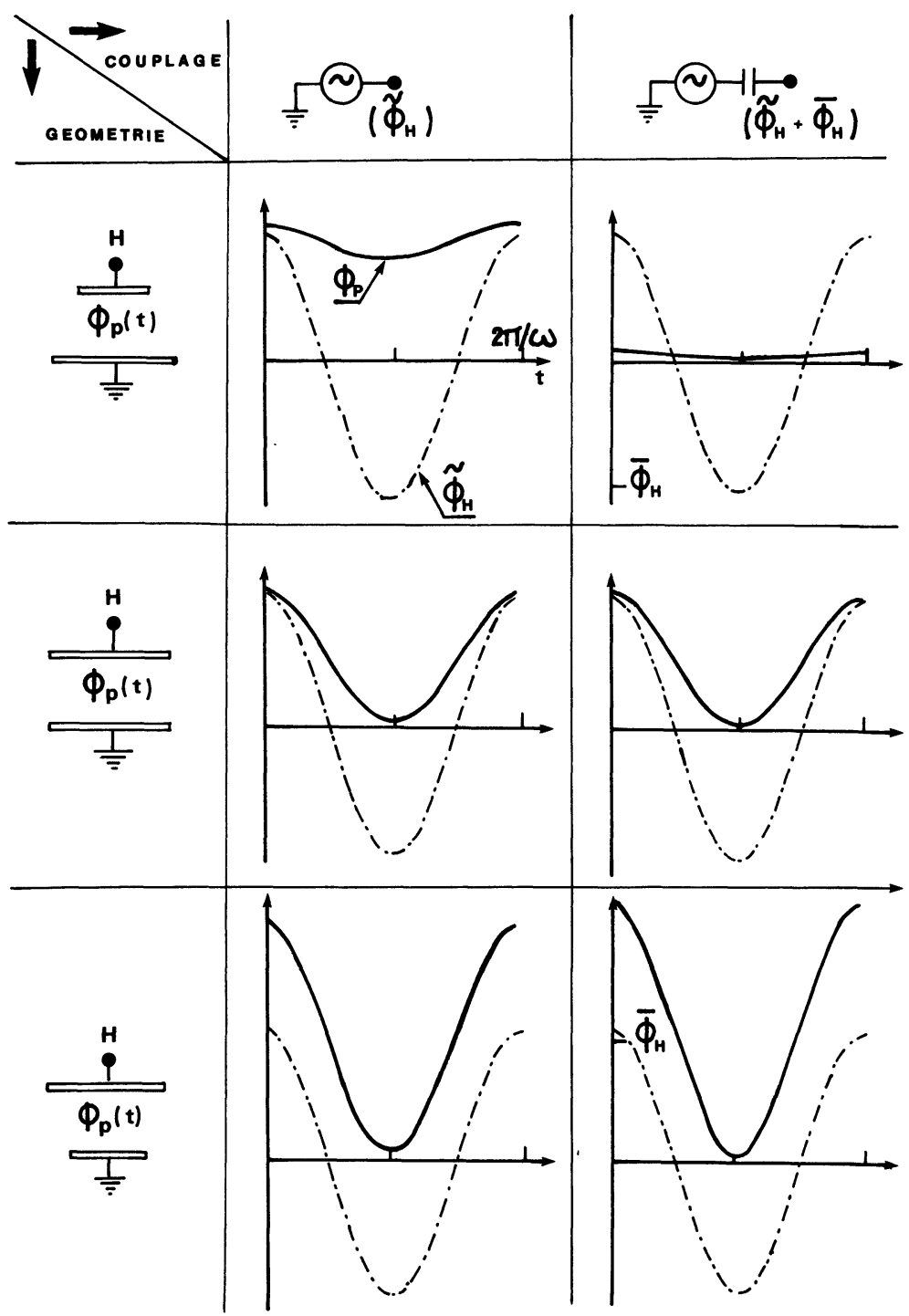

Fig. 8. - Variation temporelle du potentiel plasma (traits pleins) pour trois configurations géométriques en l'absence et en présence de capacité de blocage sur l'électrode d'excitation, $\mathrm{H}$ : cas $\omega \gg \omega_{\mathrm{pi}}$ avec $\alpha=7, V_{\mathrm{RF}}=100 k T_{\mathrm{e}} / q$, $\left|V_{\mathrm{f}}\right|=5 k T_{\mathrm{e}} / q$.

[Temporal variation of the plasma potential (solid line) for three geometrical configurations with and without a blocking capacitor on the high voltage side, $\mathrm{H}:$ case $\omega \gg \omega_{\mathrm{pi}}$, and $\alpha=7, V_{\mathrm{RF}}=100 k T_{\mathrm{e}} / q, V_{\mathrm{f}}=5 k T_{\mathrm{e}} / q$.]

pression $(p=20 \mathrm{mTorr})$. La tension d'excitation varie de 200 à $1000 \mathrm{~V}$ environ avec une fréquence comprise entre $100 \mathrm{kHz}$ et $13,6 \mathrm{MHz}$. Les mesures concernent en particulier la tension d'autopolarisation des électrodes $V_{\mathrm{DC}}$, et l'énergie $E_{\mathrm{i}}$ des ions tombant sur la grande électrode. Celle-ci est donnée sous la forme d'un spectre $g\left(E_{\mathrm{i}}\right)$, avec une valeur maximale $E_{\mathrm{iM}}$, pour les plus basses fréquences et sous la forme d'une valeur moyenne $\bar{E}_{\mathrm{i}}$, pour les plus hautes fréquences. La figure 9 présente, dans le système des coordonnées expérimentales

$$
\begin{aligned}
V_{\mathrm{DC}} & =\left(k T_{\mathrm{e}} / q\right)\left(\eta_{20}-\eta_{10}\right) \\
E_{\mathrm{iM}} & =k T_{\mathrm{e}} \eta_{1 \mathrm{M}}
\end{aligned}
$$

les points expérimentaux de la référence [6], mesurés à $105 \mathrm{kHz}$ et les courbes théoriques, calculées dans l'hypothèse quasi stationnaire pour $\alpha=7$ et pour la valeur du potentiel flottant indiquée par les auteurs. Points et courbes sont en accord raisonnable.

Pour une valeur constante de $V_{\mathrm{RF}}$, le spectre expérimental $g\left(E_{\mathrm{i}}\right)$ a été comparé au modèle du paragraphe 4.3.5 en supposant que l'analyseur en énergie a une sélectivité $\Delta E_{\mathrm{i}}$, d'où

$$
\begin{aligned}
& g_{\exp }\left(E_{\mathrm{i}}\right) \propto \frac{1}{\Delta E_{\mathrm{i}}} \times \\
& \quad \times \int_{E_{\mathrm{i}}-\Delta E_{\mathrm{i}}}^{E_{\mathrm{i}}+\Delta E_{\mathrm{i}}} \mathrm{d} E_{\mathrm{i}} /\left(1-E_{\mathrm{i}} / E_{\mathrm{iM}}\right)^{1 / 2}
\end{aligned}
$$

La figure 10a illustre cette comparaison pour $\Delta E_{\mathrm{i}} / E_{\mathrm{iM}}=0.025$. 


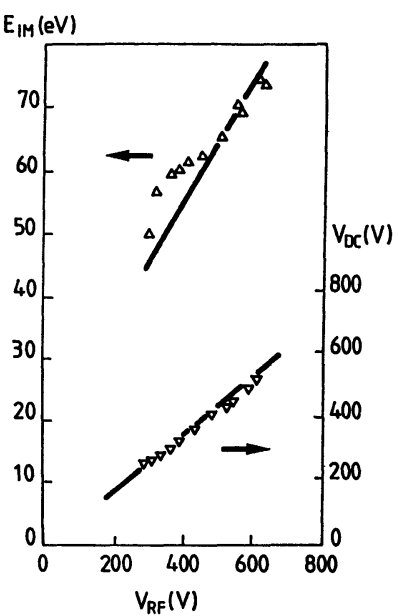

Fig. 9. - Energie maximum des ions tombant sur la grande électrode et tension d'autopolarisation en fonction de $V_{\mathrm{RF}}$. Comparaison du modèle quasi stationnaire, avec $\alpha=7$, aux points expérimentaux de la référence [6] à $105 \mathrm{kHz}$.

[Maximum energy of the ions impinging on the large electrode and self-bias voltage versus $V_{\mathrm{RF}}$. Comparison of the quasi-static state model, with $\alpha=7$, with the experimental points of reference [6] at $105 \mathrm{kHz}$.]

Les mesures à 13,6 MHz, supposées correspondre au cas $\omega \gg \omega_{\text {pi }}$, doivent être confrontées au modèle du paragraphe 4.4 et, dans ce modèle, au cas correspondant à la pression de $20 \mathrm{mTorr}$. Compte tenu des courbes de la figure 1 , nous admettrons que la chute libre constitue une approximation encore raisonnable pour les tensions d'excitation utilisées.

Dans ces conditions, les valeurs mesurées de la tension d'autopolarisation, $V_{\mathrm{DC}}$, et de l'énergie des ions tombant sur la grande électrode, $\bar{E} i$, peuvent être comparées respectivement à l'expression (113) et à

$$
\bar{E}_{\mathrm{i}}=k T_{\mathrm{e}} \bar{\eta}_{1}
$$

où $\eta_{\mathrm{j}}=\eta_{\mathrm{j} 0}$ est calculé d'après les relations (105) et (106) pour la valeur de $\gamma_{\delta}(\delta=3 / 4)$ déduite de la figure $7 \mathrm{a}$.

La figure (11) présente, en fonction de la variable $V_{\mathrm{RF}}$, la comparaison des points expérimentaux de la référence [6] avec les courbes calculées pour les mêmes valeurs de paramètres qu'à $105 \mathrm{kHz}$. Cette comparaison montre, elle aussi, un bon accord.

5.1.2 La référence [17] mentionne des mesures du potentiel moyen d'une électrode par rapport au potentiel flottant, pour différentes tensions d'excitation, dans le cas d'une décharge symétrique d'hydrogène à moyenne pression $(p \sim 38 \mathrm{mTorr})$ et à valeur élevée de $\omega / 2 \pi(68 \mathrm{MHz})$. La figure 12 montre les points correspondants et les valeurs déduites des relations (105) ou (106) pour $\gamma_{\delta}=1$. Ici encore, la comparaison modèle-expérience est satisfaisante.
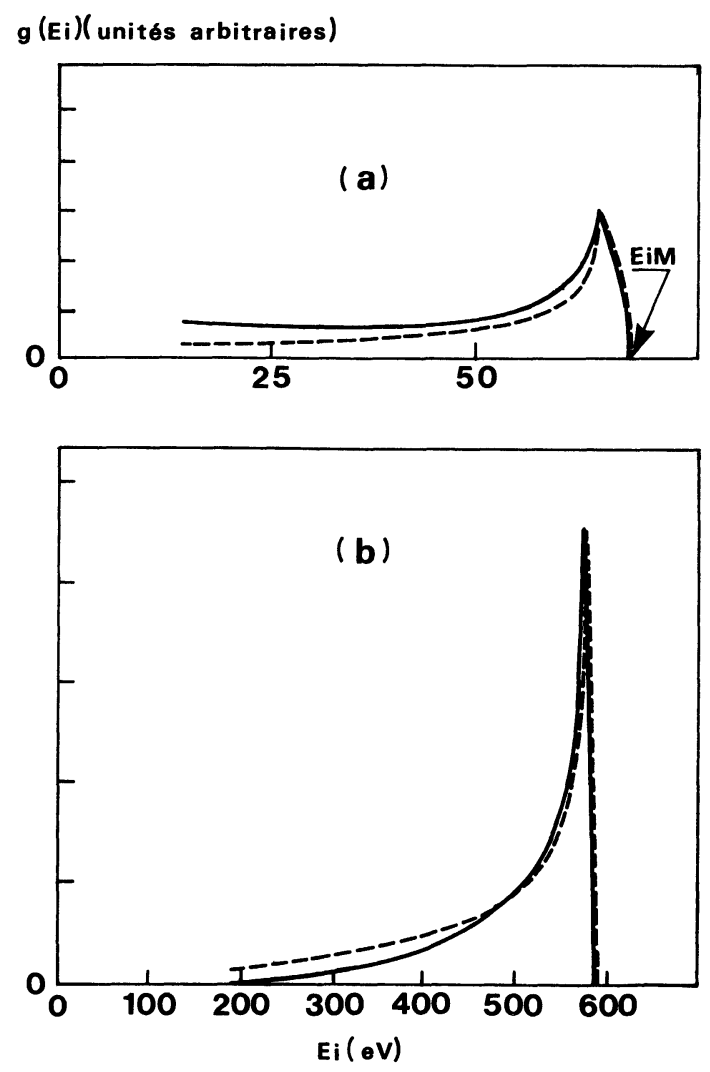

Fig. 10. - Distribution en énergie des ions tombant sur une électrode. Courbes expérimentales (traits pleins) et courbes théoriques normalisées (tiretés) pour le cas $\omega \ll \omega_{\mathrm{pi}}$. a : expérience de la référence [6] (Ar, $20 \mathrm{mTorr}$, $105 \mathrm{kHz})$. Calcul effectué avec $\Delta E_{\mathrm{i}} / E_{\mathrm{iM}}=0,025 ; \mathrm{b}$ : expérience de la référence [20] $\left(\mathrm{CF}_{4}, 10 \mathrm{mTorr}, 125 \mathrm{kHz}\right)$. Calcul effectué avec $\Delta E_{\mathrm{i}} / E_{\mathrm{i}}=1 / 70$.

[Energy distributions of the ions impinging on an electrode : experimental curves (solid lines) and normalized theoretical curves (dashed lines) for the case $\omega \ll \omega_{\mathrm{pi}}$ : a : experiment of reference [6] (Ar; $20 \mathrm{mTorr}, 105 \mathrm{kHz}$ ). Calculation made with $\Delta E_{\mathrm{i}} / E_{\mathrm{iM}}=0.025 ; \mathrm{b}$ : experiment of reference [20] $\left(\mathrm{CF}_{4}, 10 \mathrm{mTorr}, 125 \mathrm{kHz}\right)$. Calculation made with $\Delta E_{\mathrm{i}} / E_{\mathrm{i}}=1 / 70$.]

5.1.3 Le modèle du paragraphe 4.4 est en accord qualitatif avec la courbure des variations, en fonction de $\alpha$, des rapports $V_{\mathrm{DC}} / 2 V_{\mathrm{RF}}$ et $\bar{V}_{1} / \bar{V}_{2}$, mesurés à la référence [2]. La valeur de $V_{\mathrm{f}}$ n'étant pas indiquée, une comparaison quantitative précise n'est malheureusement pas possible. Par ailleurs, la référence [18] met en évidence une loi linéaire de variation de $\bar{V}_{1}$ en fonction de $\bar{V}_{2}$, du même type que la relation déduite des équations (102) et (103) :

$$
V_{1} \simeq V_{\mathrm{f}}+\bar{V}_{2} / \gamma_{\delta}
$$

Elle ne s'y compare favorablement que si la variation de $\gamma_{\delta}$ avec $V_{\mathrm{RF}}$ reste négligeable dans la plage des mesures. Ceci n'apparaît pas être le cas pour les valeurs données, confrontées aux courbes de la 


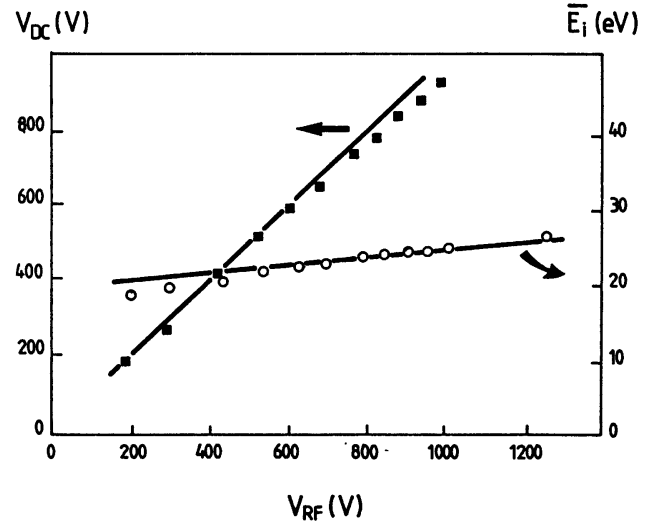

Fig. 11. - Tension d'autopolarisation et énergie moyenne des ions tombant sur la grande électrode. Comparaison du modèle à ions " gelés » avec $\alpha=7$, aux points expérimentaux de la référence [6] à $13,56 \mathrm{MHz}$.

[Self-bias voltage and mean energy of the ions impinging on the large electrode. Comparison of the «frozen » ions model with $\alpha=7$, with the experimental points of reference [6] at $13.56 \mathrm{MHz}$.]

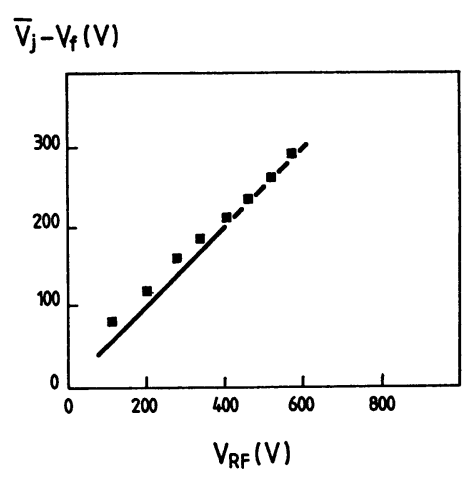

Fig. 12. - Valeur moyenne du potentiel d'une électrode, mesuré par rapport au potentiel flottant, pour une décharge symétrique. Comparaison du modèle à ions « gelés » avec des points expérimentaux à $68 \mathrm{MHz}$, d'après la référence [17].

[Electrode potential mean value measured with respect to the floating potential in a symmetrical discharge. Comparison of the «frozen » ions model with experimental points at $68 \mathrm{MHz}$, from reference [17].]

figure 7a. En outre, la pente des droites expérimentales, identifiée à $1 / \gamma_{\delta}$ correspond, d'après la figure 7a, à une valeur de $\alpha$ trop forte par un facteur d'environ 2,5. La variation observée de la pente avec $\alpha$ est cependant proche de la loi théorique $\left(\gamma_{\delta} \sim \alpha^{r}\right.$, $2<r<3$ ).

5.1.4 La référence [19] présente les mesures du potentiel plasma, effectuées avec une sonde dans une décharge RF symétrique de 300 mTorr d'Ar à $100 \mathrm{kHz}$. Ces résultats sont conformes aux variations présentées sur la partie centre-droite de la figure 6.
5.1.5 La référence [20] présente des courbes de distribution en énergie des ions des décharges RF en régime quasi statique. Certains résultats obtenus dans $\mathrm{CF}_{4}$ à 10 mTorr pour une fréquence de $125 \mathrm{kHz}$ suivent une loi proche de la relation théorique (115) pour la sélectivité en énergie $\left(\Delta E_{\mathrm{i}} / E_{\mathrm{i}} \simeq 1 / 70\right)$ donnée par les auteurs [21] (cf. Fig. 10b). Néanmoins, d'autres résultats obtenus dans la même gamme de pression et de fréquence contredisent cet accord, en particulier à $25 \mathrm{kHz}$, où des effets d'émission secondaire aux électrodes sont mis en évidence.

\subsection{COMPARAISON À D'AUTRES MODÈleS.}

5.2.1 Modèle quasi statique sans approximations. Il est intéressant de comparer le modèle présenté au paragraphe 4.3.1 au traitement complet [9] effectué dans le cadre de l'hypothèse quasi statique, traitement qui ne néglige ni le courant de déplacement, ni l'effet de la capacité de blocage, $C_{\mathrm{B}}$, sur la forme de la tension effective d'excitation entre les électrodes. Ces effets peuvent être évalués sur les figures 13 à 15.

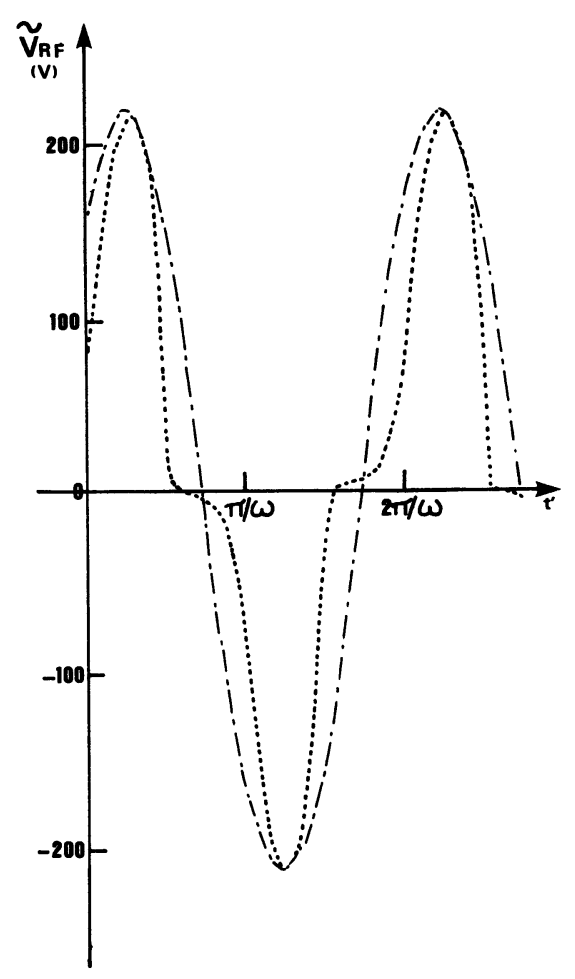

Fig. 13. - Tension effective aux bornes des électrodes d'une décharge symétrique lorsque $\omega \ll \omega_{\text {pi }}$. Variation « exacte" calculée à la référence [9] pour $\omega / 2 \pi=100 \mathrm{kHz}, C_{\mathrm{B}}=150 \mathrm{pF}$ (pointillés) et loi sinusoïdale du modèle simplifié (trait-point).

[Effective voltage across a symmetrical discharge when $\omega \ll \omega_{\text {pi }}$. «Exact » law calculated in reference [9] for $\omega / 2 \pi=100 \mathrm{kHz}, C_{\mathrm{B}}=150 \mathrm{pF}$ (dotted line) and sinusoidal law of the simplified model (dotted and dashed line).] 


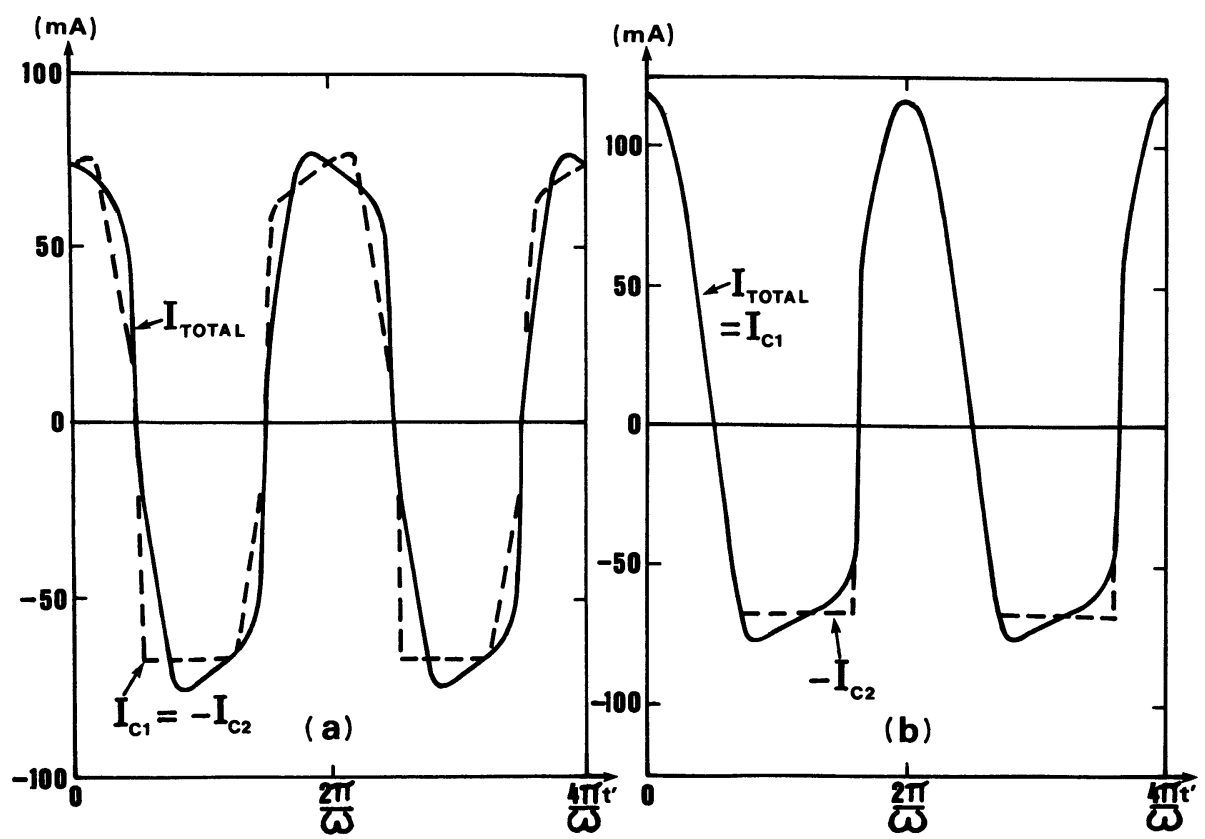

Fig. 14. - Importance du courant de déplacement dans le modèle quasi statique. Comparaison du courant de conduction sur les électrodes, $I_{\mathrm{C} 1}$ et $-I_{\mathrm{C} 2}$, avec le courant total, calculés à la référence [9] pour $\omega / 2 \pi=100 \mathrm{kHz}: \mathrm{a}$ : $\alpha=1, C_{\mathrm{B}}=150 \mathrm{pF} ; \mathrm{b}: \alpha=5, C_{\mathrm{B}}=185 \mathrm{pF}$.

[Importance of the displacement current in the quasi-static model. Comparison of the conduction current on the electrodes, $I_{\mathrm{c} 1}$ and $-I_{\mathrm{c} 2}$, with the total current, calculated in reference [9] for $\omega / 2 \pi=100 \mathrm{kHz}: \mathrm{a}: \alpha=1$, $C_{\mathrm{B}}=150 \mathrm{pF} ; \mathrm{b}: \alpha=5, C_{\mathrm{B}}=185 \mathrm{pF}$.]

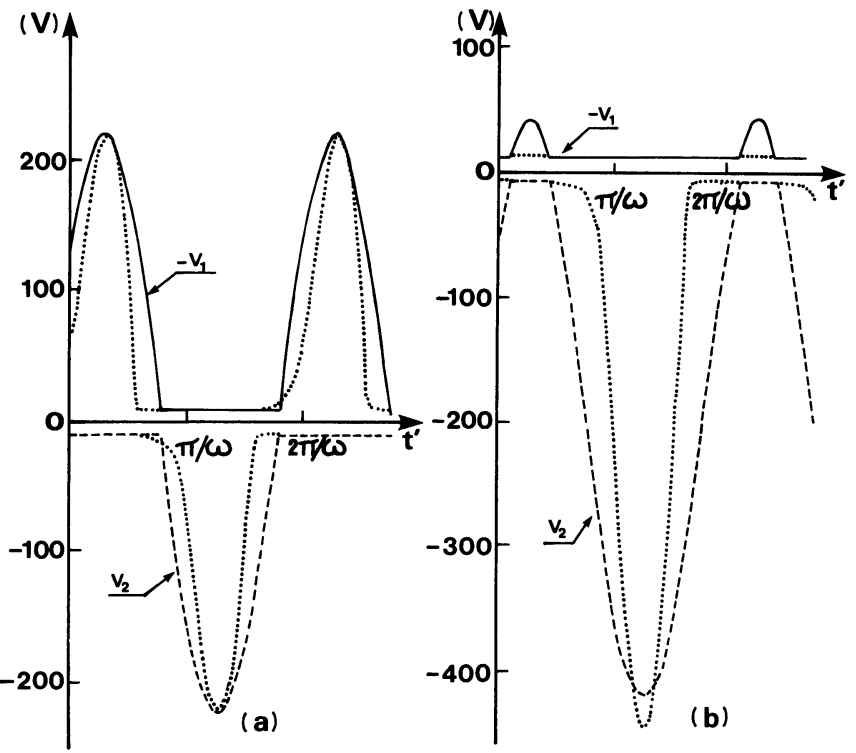

Fig. 15. - Variation temporelle de la tension des électrodes d'une décharge à $\omega \ll \omega_{\text {pi }}$. Résultats numériques « exacts » calculés à la référence [9] pour $\omega / 2 \pi=100 \mathrm{kHz}$ (pointillés) et lois de variation du modèle simplifié (tiretés et traits pleins). $a: \alpha=1$, $C_{\mathrm{B}}=150 \mathrm{pF} ; \mathrm{b}: \alpha=5, C_{\mathrm{B}}=185 \mathrm{pF}$.

[Temporal variation of the electrodes potentials in a discharge with $\omega \ll \omega_{\text {pi }}$. « Exact » numerical results calculated in reference [9] for $\omega / 2 \pi=100 \mathrm{kHz}$ (dotted line) and variation laws of the simplified model (dashed and solid line). a : $\alpha=1, C_{\mathrm{B}}=150 \mathrm{pF} ; \mathrm{b}: \alpha=5$, $C_{\mathrm{B}}=185 \mathrm{pF}$.]
L'écart de la tension RF entre électrodes par rapport à une loi sinusoïdale est montré sur la figure 13 pour le cas $\alpha=1$. Il est négligeable au voisinage des extrema de tension, mais important autour des points de tension nulle où la variation effective reste très faible pendant près de $12 \%$ de l'alternance. L'importance du courant de déplacement, $I_{\mathrm{D}_{\mathrm{j}}}$, peut être estimé sur la figure 14 qui montre l'évolution dans le temps du courant total, $I_{\text {total }}$ et des courants de conduction sur chaque électrode, $I_{\mathrm{C} 1}$ et $I_{\mathrm{C} 2}$, avec la convention de signe

$$
I_{\text {total }}=I_{\mathrm{C} 1}+I_{\mathrm{D} 1}=-\left(I_{\mathrm{C} 2}+I_{\mathrm{D} 2}\right) .
$$

Pour le cas $\alpha=1$ (Fig. 14a), l'influence de $I_{\mathrm{D}}$ est faible et n'apparaît que sur l'électrode 2 pendant une demi-alternance. Pour le cas $\alpha=5$ (Fig. 14b), il y a coïncidence exacte entre $I_{\mathrm{C} 1}$ et $-I_{\mathrm{C} 2}$, avec un écart par rapport à $I_{\text {tot }}$ qui peut être important en valeur relative mais pendant une fraction très faible de l'alternance (Fig. 15), la différence entre calculs simplifiés et calculs complets apparaît significative sur le plan quantitatif, notamment pour $\alpha=5$ (Fig. 15b).

Tout se passe en effet comme si le coefficient $\zeta$ des calculs simplifiés avait été sous-estimé par rapport à une valeur effective qui correspondrait à un rapport de surfaces des électrodes plus élevé, c'est-à-dire à une moindre contribution de la grande électrode à la d.d.p. 
Compte tenu de la discussion précédente, il semble que cette différence soit imputable essentiellement à la forme non sinusoïdale de la d.d.p. effective entre électrodes. Pour utiliser le modèle simplifié, il est donc essentiel de choisir une valeur de $C_{\mathrm{B}}$ qui n'entraîne pas de chute de potentiel notable par rapport à la d.d.p. entre électrodes. Au vu de la bonne concordance modèle-expérience du paragraphe 5.1.1, cela était sans doute le cas pour l'exemple cité.

5.2.2 Modèle quasi statique de Suzuki et al. - La référence [22] présente un modèle dont les bases physiques sont celles du modèle quasi statique du paragraphe 4.3. Les résultats, calculés pour de grandes valeurs du rapport des surfaces d'électrodes, $\alpha$, sont différents de ceux que nous avons obtenus, notamment par une loi $\mathrm{d} \bar{\eta}_{2} / \mathrm{d} \bar{\eta}_{1} \propto \alpha^{2}$, alors que le tableau I donne pour la même limite $\mathrm{d} \bar{\eta}_{2} / \mathrm{d} \bar{\eta}_{1} \simeq 2 \alpha^{3} / \pi^{2}$. La comparaison effectuée avec les mesures de la référence [18] à $13,6 \mathrm{MHz}$, qui correspondait au cas des ions gelés, ne permet pas de trancher.

5.2.3 Modèle capacitif " de Koenig ». - Le principe d'une gaine d'épaisseur fixée par la valeur moyenne du potentiel de paroi avait déjà été admis dans les modèles des décharges RF présentés aux références [1, 2 et 5]. On remarque pourtant que ces modèles conduisent à des lois de variation des potentiels d'électrode dont la dépendance, dite « loi de Koenig » en $\alpha^{4}$ ne correspond aux résultats présentés au paragraphe 4.4 que dans la limite où $V_{\mathrm{RF}} / V_{\mathrm{f}} \rightarrow \infty$. Ceci est dû au fait que ces modèles négligent la contribution du potentiel flottant $V_{\mathrm{f}}$ à la tension d'autopolarisation $V_{\mathrm{DC}}$, soit depuis le début $[1,2]$, soit plus tard dans le calcul des lois de variation [5]. La discussion, menée à la référence [23], qui utilise les résultats de la référence [18], précédemment commentés, n'apporte pas d'élément nouveau quant à la validité des différentes « loi des aires».

\section{Conclusion.}

La simplicité des modèles présentés est due à un certain nombre d'hypothèses dont la plus abrupte est, sans doute, celle qui consiste à s'affranchir des phénomènes de création de charges. Il est supposé en effet que, pour ce qui est des gaines des électrodes, tout se passe comme si l'ionisation était assurée par une source extérieure. Les modèles sont donc, par nature non autocohérents et ne prétendent pas décrire complètement la décharge. En particulier, ils passent sous silence tous les phénomènes d'émission secondaire qui pourraient se produire sur les électrodes.

D'autres hypothèses fondamentales sont celles qui permettent de décrire facilement le transport des charges au travers de la gaine et la dynamique de cette gaine. Elles impliquent des inégalités fortes, respectivement entre le libre parcours moyen et l'épaisseur de la gaine, d'une part, entre la fréquence de travail et la fréquence plasma ionique d'autre part. En outre, dans le cas des gaines collisionnelles, les mécanismes d'échange de charge n'ont pas été envisagés.

Les autres hypothèses ne sont pas strictement nécessaires mais contribuent à un calcul aisé : elles concernent l'importance relative des courants de conduction et de déplacement, et l'influence de la capacité de blocage sur la forme de la tension d'excitation effective. Elles ont été retenues dans un parti pris de simplification et aussi de généralité en ce qui concerne la dernière. Grâce à un tel traitement, il est en effet possible de mettre en évidence des lois de variation simples des potentiels d'électrode aisément utilisables dans un grand nombre d'expériences, puisqu'elles mettent en jeu des paramètres connus ou mesurables : tension sinusoïdale entre les électrodes, rapport des surfaces des électrodes, potentiel flottant, température électronique. La connaissance des autres paramètres que sont la densité électronique et la pression est a priori nécessaire pour déterminer le choix du modèle.

Dans la limite de toutes les restrictions faites précédemment et sans vouloir leur donner une portée générale, ces lois sont en bon accord avec des résultats expérimentaux publiés dans le domaine des $100 \mathrm{kHz}$ et, en basse pression, dans celui des fréquences supérieures ou égales à $13,6 \mathrm{MHz}$.

\section{Bibliographie}

[1] Koenig H. R., Maissel L. I., IBM J. Res. Dev. 14 (1970) 168.

[2] Horwitz C. M., J. Vac. Sci. Tech. A 1 (1983) 60.

[3] Van Roosmalen A. J., Van der Hoek W. G. W., Kalter H., J. Appl. Phys. 58 (1985) 653.

[4] ZAROwin C. B., J. Electrochem. Soc. 130 (1983) 1144.

[5] Keller J. H., Pennebaker W. B., IBM J. Res. Dev. 23 (1979) 3.
[6] Kohler K., Horne D. E., Coburn J. W., J. Appl. Phys. 58 (1985) 3350.

[7] GodyaK V. A., Soviet Radio Frequency Discharge Research (Delphic ass. inc., Falls Church, USA) 1986.

[8] Pointu A. M., Appl. Phys. Lett. 48 (1986) 762.

[9] Metze A., Ernie D. W., Oskam H. J., J. Appl. Phys. 60 (1986) 3081.

[10] Pointu A. M., J. Appl. Phys. 60 (1986) 4113. 
[11] Pointu A. M., Appl. Phys. Lett. 50 (1987) 1047.

[12] SwIFT J. D., Schwar M. J. R., Electric Probes for Plasmas Diagnostics (I liffe books ltd, London) 1971.

[13] Waymouth J. F., Phys. Fluids 7 (1964) 1843.

[14] Pennebaker W. B., IBM J. Res. Dev. 23 (1979) 16.

[15] Pointu A. M., Appl. Phys. Lett. 50 (1987) 316.

[16] Garscadden A., Emeleus G., Proc. Phys. Soc. 79 (1962) 535.

[17] Godyak V. A., Kuzovnikov A. A., Sov. J. Plasma Phys. 1 (1975) 276.
[18] Coburn J. W., Kay E., J. Appl. Phys. 43 (1972) 4965.

[19] Bruce R. H., J. Appl. Phys. 52 (1981) 7064.

[20] Briaud P., Turban G., Grolleau B., Mat. Res. Symp. Proc. 68 (1986) 109.

[21] Turban G., Communication privée.

[22] SuZuki K., NimomiYa K., Nishimatsu S., Thoman J. W., Steinfeld J. I., Jpn J. Appl. Phys. 25 (1986) 1569.

[23] Maniv S., J. Appl. Phys. 63 (1988) 1022. 